\title{
Hessian geometry and the holomorphic anomaly
}

\section{G.L. Cardoso ${ }^{a}$ and T. Mohaupt ${ }^{b}$}

${ }^{a}$ Center for Mathematical Analysis, Geometry and Dynamical Systems, Instituto Superior Técnico, Universidade de Lisboa, Av. Rovisco Pais, 1049-001 Lisboa, Portugal

${ }^{b}$ Department of Mathematical Sciences, University of Liverpool, Peach Street, Liverpool L69 7ZL, U.K.

E-mail: gcardoso@math.ist.ut.pt, thomas.mohaupt@liv.ac.uk

ABSTRACT: We present a geometrical framework which incorporates higher derivative corrections to the action of $N=2$ vector multiplets in terms of an enlarged scalar manifold which includes a complex deformation parameter. This enlarged space carries a deformed version of special Kähler geometry which we characterise. The holomorphic anomaly equation arises in this framework from the integrability condition for the existence of a Hesse potential.

KeYwords: Differential and Algebraic Geometry, Supergravity Models, Topological Strings

ArXiv EPrint: 1511.06658 


\section{Contents}

1 Introduction 1

2 Review of special Kähler geometry 5

2.1 Affine special Kähler manifolds 5

$\begin{array}{lll}2.2 & \text { Conical affine special Kähler manifolds } & 7\end{array}$

3 The holomorphic deformation $\quad 8$

3.1 Deformation of the immersion 8

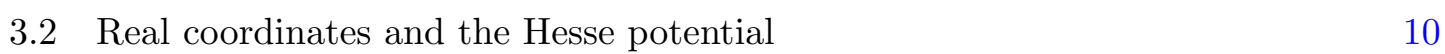

$\begin{array}{ll}3.3 \text { Deformed special Kähler geometry } & 12\end{array}$

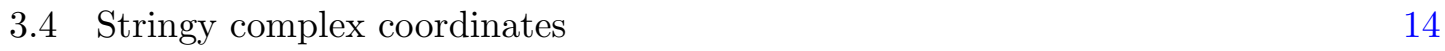

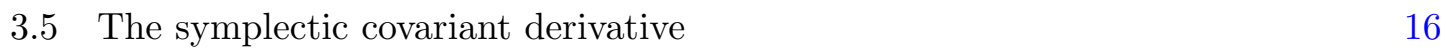

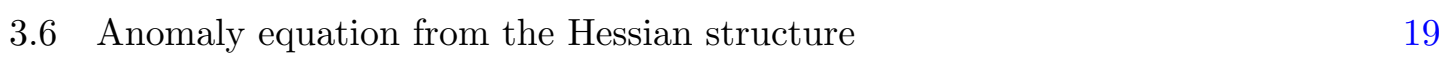

4 The non-holomorphic deformation $\quad 21$

4.1 Non-holomorphic deformation of the prepotential 21

4.2 Real coordinates and the Hesse potential 23

$\begin{array}{lll}4.3 & \text { The symplectic covariant derivative } & 25\end{array}$

$\begin{array}{ll}4.4 & \text { The holomorphic anomaly equation } \\ & 26\end{array}$

4.5 From Hessian structure to the full anomaly equation 28

$\begin{array}{llr}5 & \text { Concluding remarks } & 29\end{array}$

$\begin{array}{ll}\text { A Connections on vector bundles } & 31\end{array}$

B Special coordinates $\quad 32$

C Symplectic transformations and functions 33

\section{Introduction}

Quantum gravity is expected to manifest itself in an effective field theory framework through higher derivative terms. Supersymmetry provides some control over such terms, and in a theory of $N=2$ vector multiplets coupled to supergravity a certain class of higher derivative terms can be described by generalizing the prepotential which encodes all the couplings at the two-derivative level. While this function remains holomorphic within a Wilsonian framework, the inclusion of threshold corrections due to massless particles is known to induce non-holomorphic corrections to the couplings. In fact these corrections 
are required for consistency with electric-magnetic duality and are essential for incorporating higher derivative corrections to black hole entropy. While supergravity provides a powerful tool to organise an effective action for quantum gravity, the actual computation of couplings requires a specific theory. String theory is the natural candidate, and in particular the higher derivative corrections to $N=2$ vector multiplets are captured by the topological string. However, the relation between supergravity and the topological string is subtle, and non-holomorphic corrections are incorporated differently in the respective formalisms. In this paper we develop a new geometrical description of the higher derivative corrections on the supergravity side, by showing that they can be understood in terms of an extended scalar manifold which carries a deformed version of special geometry. We also derive various exact relations between the variables used in supergravity and in the topological string. The most interesting result we obtain is that the holomorphic anomaly equation which controls the non-holomorphic corrections in both the supergravity and topological string formalism can be derived from the integrability condition for the existence of a Hesse potential on the extended scalar manifold.

Let us next introduce our topic in more technical terms. In four dimensions, the complex scalar fields residing in $N=2$ vector multiplets parametrize a scalar manifold which is the target space of the non-linear sigma-model that enters in the Wilsonian Lagrangian describing the couplings of $N=2$ vector multiplets at the two-derivative level. The scalar manifold is an affine special Kähler manifold in global supersymmetry, and a projective special Kähler manifold in local supersymmetry; both types of target space geometry are referred to as special geometry [1-7]. Special geometry, when formulated in terms of complex variables $Y^{I}$, is encoded in a holomorphic function $F^{(0)}(Y)$, called the prepotential. When formulated in terms of special real coordinates, it is the Hesse potential that plays a central role. For affine special Kähler manifolds, the Hesse potential is related to the prepotential by a Legendre transform [8].

When coupling the $N=2$ vector multiplets to the square of the Weyl multiplet, the resulting Wilsonian Lagrangian, which now contains higher derivative terms proportional to the square of the Weyl tensor, in encoded in a generalized prepotential $F(Y, \Upsilon)$, where $\Upsilon$ denotes a complex scalar field residing in the lowest component of the square of the Weyl multiplet. The complex scalar fields $\left(Y^{I}, \Upsilon\right)$ will be called supergravity variables in the following. The prepotential $F^{(0)}(Y)$ is obtained from $F(Y, \Upsilon)$ by setting $\Upsilon=0$. Electricmagnetic duality, a central feature of $N=2$ systems based on vector multiplets, then acts by symplectic transformations of the vector $\left(Y^{I}, F_{I}\right)$, where $F_{I}=\partial F / \partial Y^{I}$. While $F(Y, \Upsilon)$ itself does not transform as a function under symplectic transformations, $F_{\Upsilon}=\partial F / \partial \Upsilon$ does [9]. The associated Hesse potential, obtained by a Legendre transform of $\operatorname{Im} F(Y, \Upsilon)$, is also a symplectic function.

Away from the Wilsonian limit, the coupling functions encoded in $F_{\Upsilon}$ receive nonholomorphic corrections, in general. In supergravity models arising from string theory, these modified coupling functions can be derived in the context of topological string theory $[10,11]$. The precise relation between these two computations is subtle, however [12]. The coupling functions computed in topological string theory depend on stringy variables $\left(\mathcal{Y}^{I}, \Upsilon\right)$ that do not coincide with the supergravity variables $\left(Y^{I}, \Upsilon\right)$ (unless $\Upsilon=0$ ). The 
precise relation between these two sets of coordinates was discussed in [12] and was used to express the supergravity Hesse potential (which is a symplectic function) in terms of stringy variables. The Hesse potential is not any longer obtained from the holomorphic generalized prepotential $F(Y, \Upsilon)$ that characterizes the Wilsonian Lagrangian. Instead, it is computed from a deformed version of $F$ that is not any longer holomorphic. It was then laboriously shown, by means of power law expansions, that the Hesse potential contains a unique subsector $\mathcal{H}^{(1)}$ that comprises coupling functions $F^{(n)}(\mathcal{Y}, \overline{\mathcal{Y}})$ that, for $n \geq 2$, satisfy the holomorphic anomaly equation of topological string theory,

$$
\frac{\partial F^{(n)}}{\partial \overline{\mathcal{Y}}^{I}}=i \bar{F}_{I}^{(0) J K}\left(\sum_{r=1}^{n-1} \partial_{J} F^{(r)} \partial_{K} F^{(n-r)}-2 \alpha D_{J} \partial_{K} F^{(n-1)}\right) .
$$

Here, $\alpha$ denotes the deformation parameter that characterizes the deviation from the Wilsonian limit. The superscript $(0)$ in $\bar{F}_{I}^{(0) J K}$ indicates that this quantity has been formed by taking derivatives of $F^{(0)}(Y)$, and that indices have been raised using the inverse $N_{(0)}^{I J}$ corresponding metric $N_{I J}^{(0)}=2 \operatorname{Im} F_{I J}^{(0)}$. If no such superscript is present, as in $\bar{F}_{I}^{J K}$, then it is understood that we take derivatives of the generalized prepotential $F(Y, \Upsilon)$, and that indices are lowered and raised using $N_{I J}=2 \operatorname{Im} F_{I J}$. This convention is applied throughout the paper.

While in topological string theory $\alpha=-\frac{1}{2}$ [10], one may ask a more general question, namely whether irrespective of the value of $\alpha$, the holomorphic anomaly equation (1.1) can be understood in terms of Hessian structures and Hessian geometry. This is indeed the case, as we will show in this paper. Namely, the anomaly equation (1.1) may be viewed as the integrability condition for the existence of a Hesse potential in supergravity. This is simplest to establish in the case when $\alpha=0$, as we will explain next (when $\alpha=0$, the coupling functions are encoded in $F_{\Upsilon}(Y, \Upsilon)$ on the supergravity side, and hence still holomorphic in supergravity variables).

Any affine special Kähler manifold $M$ can be realised as an immersion into a complex symplectic vector space $V$ [7], as we will review in section 2.1. When passing from the prepotential $F^{(0)}$ to the generalized prepotential $F(Y, \Upsilon)$, this construction gets extended, giving rise to a holomorphic family of immersions and deformed affine special Kähler manifolds, which combine into a complex manifold $\hat{M}=M \times \mathbb{C}$. By pulling back the standard Hermitian form of $V$, the space $\hat{M}$ becomes equipped with a Kähler metric $g$ and a flat torsion free connection $\nabla$ which we use to define special real coordinates. Taking the Legendre transform of the generalized prepotential $F(Y, \Upsilon)$ as a Hesse potential, we can then define a Hessian metric $g^{H}$. When analysing the integrability condition for the existence of a Hesse potential, namely that $\nabla g^{H}$ must be a completely symmetric rank three tensor in complex coordinates, one infers the anomaly equation (1.1) with $\alpha=0$, as we will show in subsection 3.6. This anomaly equation can also be viewed as a consequence of a tension between preserving holomorphicity and symplectic covariance, as follows. We introduce stringy variables $\mathcal{Y}^{I}$, and we derive various properties of the difference $\Delta Y^{I}=\mathcal{Y}^{I}-Y^{I}$. We then express the symplectic function $F_{\Upsilon}(Y, \Upsilon)$ in terms of stringy variables. By taking multiple derivatives $\partial_{\Upsilon} \mid \mathcal{Y}$ of $F_{\Upsilon}$ we obtain symplectic functions $\partial_{\Upsilon}^{n} F_{\Upsilon} \mid \mathcal{Y}$ that we express 
back in terms of supergravity variables. Then, setting $\Upsilon=0$, we obtain the set of symplectic functions $F^{(n)}=2 i D_{\Upsilon}^{n-1} F_{\Upsilon} /\left.n !\right|_{\Upsilon=0}$ introduced in [9] that, for $n \geq 2$, satisfy the anomaly equation (1.1) with $\alpha=0$. The symplectic covariant derivative $D_{\Upsilon}$ introduced in [9] is related to $\partial \Delta Y^{I} / \partial \Upsilon$, and thus it is simply a consequence of the passage from stringy to supergravity variables. The non-holomorphicity induced by the coordinate transformation reflects the tension between holomorphicity and symplecticity, and is thus a universal feature of the deformation induced by the passage from the prepotential $F^{(0)}(Y)$ to the generalized prepotential $F(Y, \Upsilon)$.

The aforementioned Hessian structure condition (namely that $\nabla g^{H}$ is totally symmetric) gives a master equation for $F_{\Upsilon}$,

$$
\partial_{\bar{I}} D_{\Upsilon} F_{\Upsilon}=\bar{F}_{I}^{J K} F_{\Upsilon J} F_{\Upsilon K},
$$

which, upon applying $D_{\Upsilon}^{n}$ to it and setting $\Upsilon=0$, yields, by induction, the anomaly equation (1.1) with $\alpha=0$ for the functions $F^{(n)}$ defined above. This master equation for $F_{\Upsilon}$ is on par with the one derived for the topological free energy $F_{\text {top }}$,

$$
F_{\text {top }}(\mathcal{Y}, \overline{\mathcal{Y}}, Q)=\sum_{n=1}^{\infty} Q^{n} F^{(n)}(\mathcal{Y}, \overline{\mathcal{Y}})
$$

where $Q$ is an expansion parameter related to the topological string coupling, which satisfies

$$
\partial_{\bar{I}} F_{\text {top }}=i \bar{F}_{I}^{(0) J K} \partial_{J} F_{\text {top }} \partial_{K} F_{\text {top }} .
$$

Next, let us discuss the case $\alpha \neq 0$. When turning on $\alpha, F_{\Upsilon}$ ceases to be holomorphic. Thus, starting from a non-holomorphic generalized prepotential $F$ as in [12], we investigate the consequences for the master equation for $F_{\Upsilon}$ that result from the Hessian structure condition. The equation we obtain is quite complicated. To compare it with (1.1), we specialise to a particular deformation, proportional to $\alpha N_{(0)}^{I J}$, where $N_{(0)}^{I J}$ is the inverse of $N_{I J}^{(0)}=-i\left(F_{I J}^{(0)}-\bar{F}_{I J}^{(0)}\right)$. Working at lowest order, we show that when setting $\Upsilon=0$, the master equation for $F_{\Upsilon}$ equals the anomaly equation (1.1) with $n=2$. The anomaly equation for the higher $F^{(n)}$ can, in principle, be obtained from this master equation by acting with multiple covariant derivatives $D_{\Upsilon}$ on it. Here, $D_{\Upsilon}$ denotes the symplectic covariant derivative introduced in [13], which is based on a non-holomorphic generalized prepotential $F$. We note that while the specific $\alpha$-deformation we picked is tied to the topological string, the framework presented in this paper is quite general and can be applied to other deformed systems, such as those discussed in [13].

The paper is organised as follows. In section 2 we review the extrinsic construction of special Kähler manifolds through immersion into a model vector space. In section 3 we deform this construction by passing from the prepotential $F^{(0)}$ to the holomorphic generalized prepotential $F(Y, \Upsilon)$. We introduce the Hessian structure based on special real coordinates, and use the latter to introduce stringy variables $\left(\mathcal{Y}^{I}, \Upsilon\right)$, as in [12]. We relate the difference $\partial \Delta Y^{I} / \partial \Upsilon$ to the symplectic covariant derivative of [9], which we subsequently use to derive a master equation for $F_{\Upsilon}$. Next, we use the Hessian structure to derive a 
different equation for $F_{\Upsilon}$, which we then relate to the holomorphic anomaly equation (1.1) with $\alpha=0$. In section 4 we redo the analysis, but now based on a non-holomorphic generalized prepotential $F$. In the concluding section we compare the approach of [12] for obtaining the holomorphic anomaly equation with the approach taken here. In the appendices we have collected some standard material to facilitate the reading of the paper.

\section{Review of special Kähler geometry}

\subsection{Affine special Kähler manifolds}

We start by reviewing the intrinsic definition of (affine) special Kähler geometry given in [6]: a Kähler manifold $(M, g, \omega)$ with complex structure $J$ is affine special Kähler if there exists a flat, torsion-free, symplectic connection $\nabla$ such that

$$
d_{\nabla} J=0
$$

We will refer to $\nabla$ as the special connection. Our convention for the relation between metric $g$, Kähler form $\omega$ and complex structure $J$ is

$$
\omega(\cdot, \cdot)=g(\cdot, J \cdot)
$$

or, in local coordinates

$$
\omega_{a c}=g_{a b} J_{c}^{b} .
$$

The definition of the exterior covariant derivative $d_{\nabla}$ is reviewed in appendix A. As shown in appendix B, in $\nabla$-affine coordinates $q^{a}$ the condition (2.1) becomes

$$
\partial_{[a} J_{c]}^{b}=0
$$

while the coefficients $\omega_{a b}$ are constant. This in turn implies that

$$
\partial_{a} g_{b c}=\partial_{b} g_{a c}
$$

which by applying the Poincaré lemma twice shows that the Kähler metric is Hessian,

$$
g_{a b}=\partial_{a, b}^{2} H,
$$

where the real function $H$ is called a Hesse potential. The coordinate-free version of this local definition of a Hessian metric is as follows: given a Riemannian metric $g$ and a flat, torsion-free connection $\nabla$, the pair $(g, \nabla)$ is called a Hessian structure, and $g$ is called a Hessian metric, if the rank-3 tensor $\nabla g$ is totally symmetric. It is easy to see that, given a Kähler manifold with a flat, torsion-free, symplectic connection $\nabla$, the condition (2.1) is equivalent to the requirement that the metric $g$ is Hessian (that is $\nabla g$ is totally symmetric).

On an affine special Kähler manifold one can choose the $\nabla$-affine coordinates $\left(q^{a}\right)=$ $\left(x^{I}, y_{I}\right)$ to be Darboux coordinates, i.e. such that the Kähler form takes the standard form

$$
\omega=2 d x^{I} \wedge d y_{I}=\Omega_{a b} d q^{a} \wedge d q^{b}, \quad\left(\Omega_{a b}\right)=\left(\begin{array}{cc}
0 & \mathbb{1} \\
-\mathbb{1} & 0
\end{array}\right) .
$$


The coordinates $q^{a}$ are called special real coordinates, and are unique up to affine transformations with symplectic linear part. ${ }^{1}$

As shown in [6] the above definition is equivalent to the well known alternative definition in terms of special holomorphic coordinates $Y^{I}$ and of a holomorphic prepotential $F\left(Y^{I}\right)$. We will now review the holomorphic formulation of special Kähler geometry in the context of the universal extrinsic construction of [7], which allows to realize any affine special Kähler manifold, at least locally. For simply connected affine special Kähler manifold this construction in fact works globally. The universal construction realises special Kähler manifolds $M$ as immersions into the standard complex symplectic vector space $V=T^{*} \mathbb{C}^{n}$, where $\operatorname{dim} V=2 \operatorname{dim} M=4 n$. We now review some details, which we are going to use for our later generalised construction.

Let $\left(Y^{I}, W_{I}\right)$ be complex Darboux coordinates on $V=T^{*} \mathbb{C}^{n} \simeq \mathbb{C}^{2 n}$. Then

$$
\Omega=d Y^{I} \wedge d W_{I}
$$

is the standard complex symplectic form on $V$, and

$$
\gamma_{V}=i \Omega\left(\cdot,{ }^{-}\right)=i\left(d Y^{I} \otimes d \bar{W}_{I}-d W_{I} \otimes d \bar{Y}^{I}\right)=g_{V}+i \omega_{V}
$$

is the associated Hermitian form, $g_{V}$ is a flat (indefinite) Kähler metric, and $\omega_{V}$ the corresponding Kähler form.

Next, let

$$
\phi: M \rightarrow V
$$

be a non-degenerate, holomorphic, Lagrangian immersion of a complex manifold $M$ of (real) dimension $2 n$ into $V$. We can assume, without loss of generality, that the image $\phi(M)$ is realized as a graph, that is the immersion has been chosen such that, when identifying $M$ locally with its image, we can take $Y^{I}$ as coordinates on the locally embedded $M$, so that in terms of coordinates $\left(Y^{I}, W_{I}\right)$ the immersion takes the form

$$
\phi: M \rightarrow V, \quad\left(Y^{I}\right) \mapsto\left(Y^{I}, F_{I}(Y)\right) .
$$

This situation is generic, and can always be achieved, at least locally, by a symplectic transformation. Since the immersion $\phi$ is Lagrangian, we have $\phi^{*} \Omega=0$, which is readily seen to be the integrability condition for the existence of a holomorphic function $F$ such that $F_{I}=\partial F / \partial Y^{I}$. In the non-generic situation where $\phi(M)$ is not realized as a graph, the immersion is still well defined and can be described using a complex symplectic vector $\left(Y^{I}(Z), W_{I}(Z)\right)$, where $Z=\left(Z^{I}\right)$ are holomorphic coordinates on $M$. However, the components $Y^{I}$ cannot be used as coordinates on $M$, and the components $W_{I}$ fail to satisfy the integrability condition for the existence of a prepotential. This is well known in the literature as a symplectic vector (or 'holomorphic section') 'without prepotential' [14]. We will assume in the following that we are in a generic symplectic frame where a prepotential exists.

\footnotetext{
${ }^{1}$ We remark that the special coordinates $\left(q^{a}\right)$ differ from standard Darboux coordinates by a conventional normalization factor, see appendix B for details.
} 
Since the immersion is non-degenerate, the pull back $\gamma_{M}=\phi^{*} \gamma_{V}$ of the Hermitian form $\gamma_{V}$ to $M$ is a non-degenerate Hermitian form, which by decomposition into real and imaginary part defines a non-degenerate metric and two-form:

$$
\gamma_{M}=g_{M}+i \omega_{M}
$$

The explicit form of $\gamma_{M}$ is

$$
\gamma_{M}=N_{I J} d Y^{I} \otimes d \bar{Y}^{J}
$$

where

$$
N_{I J}=2 \operatorname{Im} F_{I J}=-i\left(F_{I J}-\bar{F}_{I J}\right)=\frac{\partial^{2}}{\partial Y^{I} \partial \bar{Y}^{J}}\left[i\left(Y^{K} \bar{F}_{K}-F_{K} \bar{Y}^{K}\right)\right] .
$$

From this expression for $N_{I J}$ it is manifest that the metric $g_{M}$ is Kähler, with Kähler potential

$$
K=i\left(Y^{I} \bar{F}_{I}-F_{I} \bar{Y}^{I}\right),
$$

and affine special Kähler with prepotential $F$.

Special holomorphic and special real coordinates are related as follows. Given special holomorphic coordinates $Y^{I}$ on $M$, the corresponding special real coordinate are given by the real part of the complex symplectic vector $\left(Y^{I}, F_{I}\right)$ :

$$
\begin{gathered}
Y^{I}=x^{I}+i u^{I}(x, y), \\
F_{I}=y_{I}+i v_{I}(x, y) .
\end{gathered}
$$

Moreover, the holomorphic prepotential and the Hesse potential are related by a Legendre transform [8]:

$$
H(x, y)=2 \operatorname{Im} F(x+i u(x, y))-2 y_{I} u^{I}(x, y) .
$$

We remark that special real coordinates are well defined, at least locally, in any symplectic frame (including those without a prepotential) as a consequence of the non-degeneracy of the symplectic form. For simply connected special Kähler manifolds they are even globally defined functions, since the immersion is global, though not necessary global coordinates, since the immersion need not be an embedding.

\subsection{Conical affine special Kähler manifolds}

While affine special Kähler manifolds are the scalar manifolds of generic rigid $N=2$ vector multiplets, conical affine special Kähler manifolds are the scalar manifolds of rigid superconformal vector multiplets. These are in turn the starting point for the construction of the coupling of vector multiplets to Poincaré supergravity, which proceeds as follows: ${ }^{2}$ (i) start with a theory of $n+1$ superconformal vector multiplets, (ii) gauge the superconformal algebra; this introduces various connections which reside in the Weyl multiplet, (iii) partially gauge fix the superconformal transformations to obain a theory of $n$ vector multiplets coupled to Poincaré supergravity. In this construction the projective special Kähler manifold $\bar{M}$ of the supergravity theory arises as a Kähler quotient of a conical affine special

\footnotetext{
${ }^{2}$ This is reviewed in $[15,16]$.
} 
Kähler manifold. Since we will not use this construction, we refer the interested reader to the literature.

The additional condition implied by superconformal symmetry is, in terms of special holomorphic coordinates, that the prepotential is homogeneous of degree two under complex scale transformations,

$$
F\left(\lambda X^{I}\right)=\lambda^{2} F\left(X^{I}\right), \quad \lambda \in \mathbb{C}^{*}
$$

This is equivalent to the statement that the Hesse potential is homogeneous of degree two under real scale transformations of the special real coordinates, and invariant under the $\mathrm{U}(1)$ part of $\mathbb{C}^{*}$. The condition can also be formulated in a coordinate-free way [7]: a conical affine special Kähler manifold ${ }^{3}$ is a special Kähler manifold equipped with a homothetic Killing vector field $\xi$ satisfying

$$
\nabla \xi=D \xi=\mathrm{Id}
$$

where $\nabla$ is the special connection, $D$ the Levi-Civita connection, and Id the identity endomorphism on $T M$. One can then show that this implies the existence of an infinitesimal holomorphic homothetic $\mathbb{C}^{*}$ action on $M$, which is generated by $\xi$ and $J \xi$. To obtain a projective special Kähler manifold by a Kähler quotient, one needs to assume that this group action is free and proper.

\section{The holomorphic deformation}

\subsection{Deformation of the immersion}

One possible deformation of the vector multiplet action is to give it an explicit dependence on a background chiral multiplet [9], see [15] for a review. By identifying this chiral multiplet with the Weyl multiplet $W^{2}$, one can describe a particular class of higher derivative terms. Compatibility with superconformal symmetry determines the scaling behaviour of the chiral multiplet, while insisting on a local supersymmetric action implies that the dependence is holomorphic, that is the standard F-term vector multiplet action is deformed by allowing the prepotential, as a function on superspace, to depend explicitly on the chiral multiplet. After integration over superspace, the action is a local functional of the fields, which contains additional terms involving holomorphic derivatives of the prepotential with respect to the background. When identifying the chiral multiplet with the Weyl multiplet $W^{2}$, one finds that the auxiliary fields cannot any longer be eliminated in closed form, but only iteratively, thus generating an expansion in derivatives. Such an action is naturally interpreted as a Wilsonian effective action.

In the following we will investigate how the introduction of a background field can be interpreted as a deformation of special geometry. Since we focus on the scalar geometry, the background chiral field enters through its lowest component, a complex scalar denoted

\footnotetext{
${ }^{3}$ Apart from 'conical' the term 'conic' is also use in the literature.
} 
$\Upsilon$. The generalized prepotential $F(Y, \Upsilon)$ is holomorphic in $Y^{I}$ and $\Upsilon$, and (graded) homogeneous of degree two, that is

$$
F\left(\lambda Y, \lambda^{w} \Upsilon\right)=\lambda^{2} F(Y, \Upsilon), \quad \lambda \in \mathbb{C},
$$

where $w$ is the weight of $\Upsilon$ under scale transformations. If $\Upsilon$ is the lowest component of the Weyl multiplet $W^{2}$, then $w=2$. Our geometric model for the deformation parameterized by $\Upsilon$ is a map

$$
\phi: \hat{M}:=M \times \mathbb{C} \rightarrow V, \quad\left(Y^{I}, \Upsilon\right) \mapsto\left(Y^{I}, F_{I}(Y, \Upsilon)\right)
$$

which can be interpreted as a holomorphic family of immersions $\phi_{\Upsilon}: M \rightarrow V, \quad\left(Y^{I}\right) \mapsto$ $\left(Y_{I}, F_{I}(Y, \Upsilon)\right)$, that define a family of affine special Kähler structures on $M$. While $\Upsilon$ is a scalar under symplectic transformations, it enters into the transformation of the complex symplectic vector $\left(Y^{I}, F_{I}(Y, \Upsilon)\right)$, and other objects, through the generalized prepotential. Our set-up is consistent with [9], in particular we can draw on the various formulae for symplectic transformations derived there.

We define a metric and a two-form on $\hat{M}=M \times \mathbb{C}$ by pulling back the canonical hermitian form $\gamma_{V}$ :

$$
\gamma=\phi^{*} \gamma_{V}=g+i \omega=N_{I J} d Y^{I} \otimes d \bar{Y}^{J}+i \bar{F}_{I \Upsilon} d Y^{I} \otimes d \bar{\Upsilon}-i F_{I \Upsilon} d \Upsilon \otimes d \bar{Y}^{I}
$$

where $N_{I J}=-i\left(F_{I J}-\bar{F}_{I J}\right)$. We assume that $\gamma$ is non-degenerate, which certainly is true for sufficiently small $\Upsilon{ }^{4}$ In the following, holomorphic coordinates on $\hat{M}$ are denoted $\left(v^{A}\right)=\left(Y^{I}, \Upsilon\right)$. Using the conventions

$$
\begin{aligned}
d a d b & =\frac{1}{2}(d a \otimes d b+d b \otimes d a), \\
d a \wedge d b & =d a \otimes d b-d b \otimes d a,
\end{aligned}
$$

we obtain the metric

$$
g=g_{A B} d v^{A} d \bar{v}^{B}=N_{I J} d Y^{I} d \bar{Y}^{J}+i \bar{F}_{I \Upsilon} d Y^{I} d \bar{\Upsilon}-i F_{J \Upsilon} d \Upsilon d \bar{Y}^{J}
$$

which is a Kähler metric $g_{A B}=\partial_{A, \bar{B}}^{2} K$ with Kähler potential

$$
K=-i\left(\bar{Y}^{I} F_{I}(Y, \Upsilon)-\bar{F}_{I}(\bar{Y}, \bar{\Upsilon}) Y^{I}\right)
$$

and

$$
\omega=-\frac{i}{2} N_{I J} d Y^{I} \wedge d \bar{Y}^{J}+\frac{1}{2} \bar{F}_{I \Upsilon} d Y^{I} \wedge d \bar{\Upsilon}-\frac{1}{2} F_{I \Upsilon} d \Upsilon \wedge d \bar{Y}^{I}
$$

is the associated Kähler form. The Kähler metric $g_{A B}$ has occured in the deformed sigma model [17], which provides a field theoretic realization of our set-up.

\footnotetext{
${ }^{4}$ In applications $\Upsilon$ will not necessarily be small, but it is reasonable to expect that $\gamma$ is non-degenerate, at least generically.
} 


\subsection{Real coordinates and the Hesse potential}

Following [18], we now define special real coordinates and a Hesse potential in presence of the deformation. Special real coordinates are defined by

$$
Y^{I}=x^{I}+i u^{I}(x, y, \Upsilon, \bar{\Upsilon}), \quad F_{I}=y_{I}+i v_{I}(x, y, \Upsilon, \bar{\Upsilon})
$$

and the (generalized) Hesse potential is related to the (generalized) prepotential by a Legendre transform:

$$
H(x, y, \Upsilon, \bar{\Upsilon})=-i(F-\bar{F})-2 y_{I} u^{I}(x, y, \Upsilon, \bar{\Upsilon})
$$

where $F=F(Y(x, u(x, y, \Upsilon, \bar{\Upsilon})), \Upsilon)$.

We are interested in the coordinate transformation between special complex and special real coordinates ${ }^{5}$

$$
(x, u, \Upsilon, \bar{\Upsilon}) \mapsto(x, y(x, u, \Upsilon, \bar{\Upsilon}), \Upsilon, \bar{\Upsilon})
$$

and its inverse

$$
(x, y, \Upsilon, \bar{\Upsilon}) \mapsto(x, u(x, y, \Upsilon, \bar{\Upsilon}), \Upsilon, \bar{\Upsilon})
$$

When rewriting derivatives between the coordinate systems, one needs to carefully use the chain rule: when differentiating a function $f=f(x, y(x, u, \Upsilon, \bar{\Upsilon}), \Upsilon, \bar{\Upsilon})$ the following formulae are useful

$$
\begin{aligned}
\left.\frac{\partial f}{\partial x^{I}}\right|_{u} & =\left.\frac{\partial f}{\partial x^{I}}\right|_{y}+\left.\frac{\partial f}{\partial y_{K}}\right|_{x} \frac{\partial y_{K}}{\partial x^{I}}, \\
\left.\frac{\partial f}{\partial u^{I}}\right|_{x} & =\left.\frac{\partial f}{\partial y_{K}}\right|_{x} \frac{\partial y_{K}}{\partial u^{I}}, \\
\left.\frac{\partial f}{\partial \Upsilon}\right|_{x, u} & =\left.\frac{\partial f}{\partial \Upsilon}\right|_{x, y}+\left.\frac{\partial f}{\partial y_{K}}\right|_{x} \frac{\partial y_{K}}{\partial \Upsilon} .
\end{aligned}
$$

The Jacobians for the coordinate transformations take the form

$$
\frac{D(x, u, \Upsilon, \bar{\Upsilon})}{D(x, y, \Upsilon, \bar{\Upsilon})}=\left(\begin{array}{cccc}
\mathbb{1} & 0 & 0 & 0 \\
\left.\frac{\partial u}{\partial x}\right|_{y} & \left.\frac{\partial u}{\partial y}\right|_{x} & \left.\frac{\partial u}{\partial \Upsilon}\right|_{x, y} & \left.\frac{\partial u}{\partial \widetilde{\Upsilon}}\right|_{x, y} \\
0 & 0 & \mathbb{1} & 0 \\
0 & 0 & 0 & \mathbb{1}
\end{array}\right)
$$

and

$$
\frac{D(x, y, \Upsilon, \bar{\Upsilon})}{D(x, u, \Upsilon, \bar{\Upsilon})}=\left(\begin{array}{cccc}
\mathbb{1} & 0 & 0 & 0 \\
\left.\frac{\partial y}{\partial x}\right|_{u} & \left.\frac{\partial y}{\partial u}\right|_{x} & \left.\frac{\partial y}{\partial \Upsilon}\right|_{x, u} & \left.\frac{\partial y}{\partial \Upsilon}\right|_{x, u} \\
0 & 0 & \mathbb{1} & 0 \\
0 & 0 & 0 & \mathbb{1}
\end{array}\right)
$$

\footnotetext{
${ }^{5}$ We find it convenient to work with $\Upsilon$ and $\bar{\Upsilon}$ when using special 'real' coordinates instead of decomposing them into their real and imaginary parts.
} 
By the chain rule it is straightforward to evaluate

$$
\frac{D(x, y, \Upsilon, \bar{\Upsilon})}{D(x, u, \Upsilon, \bar{\Upsilon})}=\left(\begin{array}{cccc}
\mathbb{1} & 0 & 0 & 0 \\
\frac{1}{2} R & -\frac{1}{2} N & \frac{1}{2} F_{I \Upsilon} & \frac{1}{2} \bar{F}_{I \Upsilon} \\
0 & 0 & \mathbb{1} & 0 \\
0 & 0 & 0 & \mathbb{1}
\end{array}\right)
$$

where $2 F_{I J}=R_{I J}+i N_{I J}$. This matrix can easily be inverted, with the result:

$$
\frac{D(x, u, \Upsilon, \bar{\Upsilon})}{D(x, y, \Upsilon, \bar{\Upsilon})}=\left(\begin{array}{cccc}
\mathbb{1} & 0 & 0 & 0 \\
N^{-1} R & -2 N^{-1} & N^{-1} F_{I \Upsilon} & N^{-1} \bar{F}_{I \Upsilon} \\
0 & 0 & \mathbb{1} & 0 \\
0 & 0 & 0 & \mathbb{1}
\end{array}\right)
$$

In order to transform the Kähler metric to special real coordinates, the following relations are useful:

$$
\frac{\partial H}{\partial x^{I}}=2 v_{I}, \quad \frac{\partial H}{\partial y_{I}}=-2 u^{I} .
$$

Moreover, using the chain rule one computes:

$$
\begin{aligned}
& \left.\frac{\partial v_{I}}{\partial x^{J}}\right|_{y}=\frac{1}{2}\left(N+R N^{-1} R\right)_{I J}, \\
& \left.\frac{\partial v_{I}}{\partial y_{J}}\right|_{x}=-\left.\frac{\partial u^{J}}{\partial x^{I}}\right|_{y}=2\left(N^{-1}\right)^{I J}, \\
& \left.\frac{\partial v_{I}}{\partial u^{J}}\right|_{x}=\frac{1}{2} R_{I J} .
\end{aligned}
$$

Using the notation $\left(q^{a}\right)=\left(x^{I}, y_{I}\right)$, the Kähler metric $g$ expressed in special real variables takes the form

$$
g=\frac{\partial^{2} H}{\partial q^{a} \partial q^{b}} d q^{a} d q^{b}+\frac{\partial^{2} H}{\partial q^{a} \partial \Upsilon} d q^{a} d \Upsilon+\frac{\partial^{2} H}{\partial q^{a} \partial \bar{\Upsilon}} d q^{a} d \bar{\Upsilon},
$$

where

$$
\left(\frac{\partial^{2} H}{\partial q^{a} \partial q^{b}}\right)=\left(\begin{array}{cc}
N+R N^{-1} R & -2 R N^{-1} \\
-2 N^{-1} R & 4 N^{-1}
\end{array}\right)
$$

and

$$
\begin{aligned}
\frac{\partial^{2} H}{\partial x^{I} \partial \Upsilon} & =2 \bar{F}_{I M} N^{M N} F_{N \Upsilon}, & \frac{\partial^{2} H}{\partial x^{I} \partial \bar{\Upsilon}} & =2 F_{I M} N^{M N} \bar{F}_{N \Upsilon}, \\
\frac{\partial^{2} H}{\partial y_{I} \partial \Upsilon} & =-2 N^{I J} F_{J \Upsilon}, & \frac{\partial^{2} H}{\partial y_{I} \partial \bar{\Upsilon}} & =-2 N^{I J} \bar{F}_{J \Upsilon} .
\end{aligned}
$$

In the undeformed case the Kähler metric is simultaneously Hessian. To see whether this is still the case, we first note that $\hat{M}$ can be equipped with an affine structure and thus a Hessian metric $g^{H}$ with Hesse potential $H$ can be defined. This requires the existence of a flat, torsion-free connection. For fixed $\Upsilon$ we know that the special connection $\nabla$ is 
such a connection, with affine coordinates $x^{I}, y_{I}$. We can extend $\nabla$ to a flat, torsion-free connection on $\hat{M}=M \times \mathbb{C}$ by imposing

$$
\nabla d x^{I}=0, \quad \nabla d y_{I}=0, \quad \nabla d \Upsilon=0, \quad \nabla d \bar{\Upsilon}=0 .
$$

If $x^{I}, y_{I}$ are not global coordinates on $M$, we use that $M$ can be covered by special real coordinate systems, which are related by affine transformations with symplectic linear part. Since for fixed $\Upsilon \neq 0$ the map $\phi_{\Upsilon}$ still induces an affine special Kähler structure, special real coordinate systems extend to $\hat{M}$ and provide it with the affine structure required to define a flat torsion-free connection.

Upon computing the components of the Hessian metric $g^{H}$ explicitly, we realize that is not equal to the Kähler metric $g$. The difference between the two metrics is

$$
g^{H}-g=\partial^{2} H_{\mid x, y}=\frac{\partial^{2} H}{\partial \Upsilon \partial \Upsilon} d \Upsilon d \Upsilon+2 \frac{\partial^{2} H}{\partial \Upsilon \partial \bar{\Upsilon}} d \Upsilon d \bar{\Upsilon}+\frac{\partial^{2} H}{\partial \bar{\Upsilon} \partial \bar{\Upsilon}} d \bar{\Upsilon} d \bar{\Upsilon},
$$

where

$$
\begin{aligned}
& \frac{\partial^{2} H}{\partial \Upsilon \partial \bar{\Upsilon}}=N^{I J} F_{I \Upsilon} \bar{F}_{J \Upsilon}, \quad \frac{\partial^{2} H}{\partial \Upsilon \partial \Upsilon}=-i F_{\Upsilon \Upsilon}+N^{I J} F_{I \Upsilon} F_{J \Upsilon}, \\
& \frac{\partial^{2} H}{\partial \bar{\Upsilon} \partial \bar{\Upsilon}}=i \bar{F}_{\Upsilon \Upsilon}+N^{I J} \bar{F}_{I \Upsilon} \bar{F}_{J \Upsilon} .
\end{aligned}
$$

We remark that these metric coefficients are symplectic functions, see [9], which is necessary in order that $g^{H}-g$ is a well defined tensor field (which we know to be the case, because $g^{H}$ and $g$ are both metric tensors). We further remark that

$$
2 H=K-2 i \Upsilon F_{\Upsilon}+2 i \Upsilon \bar{\Upsilon} \bar{F}_{\Upsilon}
$$

differs from the Kähler potential (3.2) by a Kähler transformation. Therefore $2 H$, taken as a Kähler potential, defines the same Kähler metric $g=g^{K}$ as $K$. However, when taking $K$ as a Hesse potential one does not get the Hessian metric $g^{H}$. While a Kähler potential is unique up to Kähler transformations, a Hesse potential is unique up to affine transformations. Moreover, since our Hessian metric has definite scaling properties, we can impose that the Hesse potential is homogeneous of degree two, which is automatic in the way we have defined it as the Legendre transform of the generalized prepotential. If homogeneity is imposed on top of using special real coordinates, then the Hesse potential is unique up to symplectic transformations. We remark that the Hesse potential is the sum of two symplectic functions. Different linear combinations of these two functions define different metrics. By inspection one finds that defining the (generalized) Hesse potential as the Legendre transform of the generalized prepotential leads to a particularly simple form of the coefficients $\partial^{2} H_{\mid x, y}$. We will see later how the Hessian metric $g^{H}$ encodes the holomorphic anomaly equation.

\subsection{Deformed special Kähler geometry}

We are now in position to demonstrate that $\hat{M}$ carries itself a deformed version of affine special Kähler geometry. We have already seen that $g$ is a Kähler metric with Kähler form 
$\omega$. To compare this with the two-form $2 d x^{I} \wedge d y_{I}$, which is the Kähler form on $M$, we compute

$$
\begin{aligned}
2 d x^{I} \wedge d y_{I}= & -\frac{i}{2} N_{I J} d Y^{I} \wedge d \bar{Y}^{J}-\frac{1}{2} F_{I \Upsilon} d \Upsilon \wedge d \bar{Y}^{I}+\frac{1}{2} \bar{F}_{I \Upsilon} d Y^{I} \wedge d \bar{\Upsilon} \\
& +\frac{1}{2} F_{I \Upsilon} d Y^{I} \wedge d \Upsilon+\frac{1}{2} \bar{F}_{I \Upsilon} d \bar{Y}^{I} \wedge d \bar{\Upsilon},
\end{aligned}
$$

and therefore the Kähler form can be written as

$$
\omega=2 d x^{I} \wedge d y_{I}-\frac{1}{2} F_{I \Upsilon} d Y^{I} \wedge d \Upsilon-\frac{1}{2} \bar{F}_{I \Upsilon} d \bar{Y}^{I} \wedge d \bar{\Upsilon} .
$$

This shows in particular that $2 d x^{I} \wedge d y_{I}$, when considered as a form on $\hat{M}$, is not of type $(1,1)$ (since $\omega$ is, and both differ by pure forms). Using the rewriting

$$
F_{I \Upsilon} d Y^{I} \wedge d \Upsilon=d F_{\Upsilon} \wedge d \Upsilon=-d\left(\Upsilon d F_{\Upsilon}\right)
$$

we find

$$
\omega=2 d x^{I} \wedge d y_{I}+\frac{1}{2} d\left(\Upsilon d F_{\Upsilon}\right)+\frac{1}{2} d\left(\bar{\Upsilon} d \bar{F}_{\Upsilon}\right) .
$$

Thus the difference between the Kähler forms $\omega$ of $\hat{M}$ and $2 d x^{I} \wedge d y_{I}$ of $M$ is exact, so that both forms are homologous. The deformation involves the function $F_{\Upsilon}=\partial_{\Upsilon} F$, which plays a central role in describing the deformation and should be viewed as the supergravity counterpart of the topological free energy $F_{\text {top }}$. First, note that while the generalized prepotential $F$, and its higher derivatives $\partial_{\Upsilon}^{n} F$ with $n>1$, are not symplectic functions, $F_{\Upsilon}$ is a symplectic function [9]. Moreover, it is independent of the undeformed (twoderivative) prepotential $F^{(0)}(Y)=F(Y, \Upsilon=0)$, but contains all the information about the deformation. We remark that while within the present construction $F_{\Upsilon}$ is holomorphic, this condition will be relaxed later.

Next we compute

$$
\nabla \omega=-\frac{1}{2} d\left(F_{I \Upsilon}\right) \otimes\left(d Y^{I} \wedge d \Upsilon\right)+\text { c.c. }
$$

which shows that $\omega$ is not parallel, and the connection $\nabla$ is not a symplectic connection on $\hat{M}$. This shows that while $(\hat{M}, g, \omega, \nabla)$ is Kähler, it is not special Kähler. The deformation is controlled by an exact form, which is determined by the symplectic function $F_{\Upsilon}$.

The fourth condition on a special connection is that the complex structure is covariantly closed. To compute the exterior covariant derivative of the complex structure $J$, we note that the vector fields $\partial_{x^{I}}, \partial_{y_{I}}, \partial_{\Upsilon}, \partial_{\bar{\Upsilon}}$ define a $\nabla$-parallel frame which is dual to the $\nabla$ parallel co-frame $d x^{I}, d y_{I}, d \Upsilon, d \bar{\Upsilon}$. Using this one verifies that

$$
\nabla \frac{\partial}{\partial Y^{I}}=\nabla\left(\frac{1}{2} \frac{\partial}{\partial x^{I}}+\frac{1}{2} F_{I J} \frac{\partial}{\partial y_{J}}\right)=\frac{1}{2} d F_{I J} \otimes \frac{\partial}{\partial y_{J}} .
$$

Using that $d_{\nabla} J=d J^{a} e_{a}-J^{a} \wedge d_{\nabla} e_{a}$ where $e_{a}$ is any basis of sections of $T \hat{M}$, so that $d_{\nabla} e_{a}=\nabla e_{a}$, we find

$$
d_{\nabla} J=\left(-i d Y^{I} \wedge \frac{1}{2} d F_{I J}+\text { c.c. }\right) \otimes \frac{\partial}{\partial y_{J}} .
$$


Note the rewriting

$$
d Y^{I} \wedge d F_{I J}=d Y^{I} \wedge F_{I J \Upsilon} d \Upsilon=-d\left(F_{I J} d Y^{I}\right)=d\left(F_{I \Upsilon} d \Upsilon\right)
$$

where we used symmetry of $F_{I J}$ and the chain rule. Therefore

$$
d_{\nabla} J=\left(-i d\left(F_{I \Upsilon} d \Upsilon\right)+\text { c.c. }\right) \otimes \frac{\partial}{\partial y_{I}}=\left(-i F_{I J \Upsilon} d Y^{J} \wedge d \Upsilon+\text { c.c. }\right) \otimes \frac{\partial}{\partial y_{I}},
$$

which is non-vanishing. As a consistency check, observe that it is manifest that $d_{\nabla}^{2}=0$, which must be true because $\nabla$ is flat. Since the complex structure $J$ of $\hat{M}$ is not covariantly closed, the fourth condition required on the connection $\nabla$ in order to define a special Kähler manifold is also violated. Again the deformation involves an exact form constructed out of the function $F_{\Upsilon}$.

In summary, $(\hat{M}=M \times \mathbb{C}, J, g)$ is a Kähler manifold with Kähler form $\omega$, equipped with a flat, torsion-free connection, such that $\nabla \omega$ and $d_{\nabla} J$ are given by (3.5) and (3.6). We will call such manifolds deformed affine special Kähler manifolds. Since our definition involves the map $\phi$, this is not an intrinsic definition, but the name for a specific construction.

For completeness we remark that the pullback of the complex symplectic form $\Omega$ of $V$ is non-vanishing: ${ }^{6}$

$$
\phi^{*} \Omega=F_{I \Upsilon} d Y^{I} \wedge d \Upsilon=-d\left(\Upsilon d F_{\Upsilon}\right) .
$$

As we by now expect, the right hand side is exact and controlled by $F_{\Upsilon}$.

\subsection{Stringy complex coordinates}

The framework introduced so far is based on a generalized holomorphic prepotential $F(Y, \Upsilon)$, a complex symplectic vector $\left(Y^{I}, F_{I}(Y, \Upsilon)\right)$ and a map $\phi: \hat{M} \rightarrow V$ which introduces a Kähler metric $g$ on $\hat{M}=M \times \mathbb{C}$, which deviates from being special Kähler if $F_{\Upsilon} \neq 0$. Although $\Upsilon$ is a symplectic scalar, symplectic transformations of data derived from $F$ or the symplectic vector $\left(Y^{I}, F_{I}\right)$ depend on $\Upsilon$. If we expand $F$ in a power series

$$
F(Y, \Upsilon)=\sum_{g=0}^{\infty} F^{(g)}(Y) \Upsilon^{g}
$$

then the functions $F^{(g)}(Y)$ are holomorphic and homogeneous of degree $2-2 g$, but they are not symplectic functions, and transform in a complicated way under symplectic transformations.

When the background is identified with the Weyl multiplet $W^{2}$, our formalism describes an Wilsonian effective action for vector multiplets which includes a certain class of higher derivative terms. The same class of terms can be described using the topological string, but the formalism used in this context is different. There is no generalized prepotential, but instead one works with an undeformed complex symplectic vector $\left(\mathcal{Y}^{I}, F_{I}^{(0)}(\mathcal{Y})\right)$. The information which is encoded in the symplectic function $F_{\Upsilon}$ in the supergravity formalism

\footnotetext{
${ }^{6}$ It is of course clear already for dimensional reasons that $\hat{M}$ cannot be a (locally immersed) Lagrangian submanifold of $V$.
} 
is then differently encoded in a hierarchy of genus- $g$ topological free energies $F^{(g)}(\mathcal{Y}, \overline{\mathcal{Y}})$ which individually are symplectic functions, at the expense of not being holomorphic. The deviation from holomorphicity is controlled by the holomorphic anomaly equation. Elaborating on [12], we will now show that the relation between the two frameworks can be understood as a coordinate transformation. This will proceed in two steps. First we will show that when starting from the holomorphically deformed special geometry introduced so far, one obtains a hierarchy of free energies, where $F^{(1)}$ is holomorphic, while the $F^{(g)}$ with $g>1$ are non-holomorphic and satisfy a version of the holomorphic anomaly equation where the two-derivative term is absent. This is not quite the situation for the topological string, where already $F^{(1)}$ is non-holomorpic and the anomaly equation requires an additional twoderivative term. In the next section we will generalize our deformed special geometry by making it explicitly non-holomorphic, and then show that by a coordinate transformation we obtain the full anomaly equation.

The relation between the supergravity coordinates $Y^{I}$ and the stringy coordinates $\mathcal{Y}^{I}$ is defined by imposing that the corresponding special real coordinates agree [12]:

$$
\left(\begin{array}{c}
2 x^{I} \\
2 y_{I}
\end{array}\right)=\left(\begin{array}{c}
Y^{I}+\bar{Y}^{I} \\
F_{I}(Y, \Upsilon)+\bar{F}_{I}(\bar{Y}, \bar{\Upsilon})
\end{array}\right)=\left(\begin{array}{c}
\mathcal{Y}^{I}+\overline{\mathcal{Y}}^{I} \\
F_{I}^{(0)}(\mathcal{Y})+\bar{F}_{I}^{(0)}(\overline{\mathcal{Y}})
\end{array}\right) .
$$

This implicitly defines a non-holomorphic coordinate transformation between complex coordinates on $\hat{M}$,

$$
\left(Y^{I}, \Upsilon\right) \mapsto\left(\mathcal{Y}^{I}, \Upsilon\right)
$$

which we parametrize as [12]

$$
\mathcal{Y}^{I}=Y^{I}+\Delta Y^{I}(Y, \bar{Y}, \Upsilon, \bar{\Upsilon})
$$

Note that by construction $\mathcal{Y}^{I}=Y^{I}$ for $\Upsilon=0$. In particular, the $\mathcal{Y}^{I}$ still are holomorphic coordinates on $M$. If $\Upsilon \neq 0$ the coordinate transformation can be constructed iteratively [12]. Since this gets complicated very soon, we will focus on statements that can be made without expansion or iteration.

To this end, let us consider the two-form $2 d x^{I} \wedge d y_{I}$ given in (3.3). Using (3.7), we express this two-form in the new complex variables $\left(\mathcal{Y}^{I}, \overline{\mathcal{Y}}^{I}\right)$, obtaining

$$
\begin{aligned}
2 d x^{I} \wedge d y_{I}=-\frac{i}{2} N_{I J}[ & -\frac{\partial \Delta Y^{J}}{\partial \mathcal{Y}^{K}} d \mathcal{Y}^{K} \wedge d \mathcal{Y}^{I}-\frac{\partial \Delta Y^{J}}{\partial \overline{\mathcal{Y}}^{K}} d \overline{\mathcal{Y}}^{K} \wedge d \overline{\mathcal{Y}}^{I} \\
& +\left(\delta_{K}^{I} \delta_{L}^{J}-\frac{\partial \Delta Y^{I}}{\partial \mathcal{Y}^{K}} \delta_{L}^{J}+\delta_{K}^{I} \frac{\partial \Delta Y^{J}}{\partial \overline{\mathcal{Y}}^{L}}\right) d \mathcal{Y}^{K} \wedge d \overline{\mathcal{Y}}^{L} \\
& \left.+2 \frac{\partial \Delta Y^{I}}{\partial \Upsilon} d x^{J} \wedge d \Upsilon+2 \frac{\partial \Delta Y^{I}}{\partial \bar{\Upsilon}} d x^{J} \wedge d \bar{\Upsilon}\right] \\
& +F_{I \Upsilon} d x^{I} \wedge d \Upsilon+\bar{F}_{I \Upsilon} d x^{I} \wedge d \bar{\Upsilon}
\end{aligned}
$$

where in the last two lines we combined various terms into terms containing $d x^{I}$. We now convert all differentials appearing in (3.8) to the real flat frame $\left(d x^{I}, d y_{I}, d \Upsilon, d \bar{\Upsilon}\right)$ using

$$
d \mathcal{Y}^{I}=d x^{I}+i N_{(0)}^{I K} R_{K J}^{(0)} d x^{J}-2 i N_{(0)}^{I J} d y_{J},
$$


where here and in the following we use a notation where the subscript or superscript $(0)$ indicates that a quantity has been calculated using the undeformed prepotential $F^{(0)}(\mathcal{Y})=$ $F(Y, \Upsilon=0)$. Then, by comparing the differentials on both sides of the resulting expression, we obtain the relations

$$
\begin{aligned}
\frac{\partial \Delta Y^{J}}{\partial \Upsilon} & =-i N^{J K} F_{K \Upsilon}, \\
N^{I J} & =\left(\delta_{K}^{I}-\frac{\partial \Delta Y^{I}}{\partial \mathcal{Y}^{K}}+\frac{\partial \Delta Y^{I}}{\partial \overline{\mathcal{Y}}^{K}}\right) N_{(0)}^{K J}, \\
N_{[I K} \frac{\partial \Delta Y^{K}}{\partial \mathcal{Y}^{J]}} & =0,
\end{aligned}
$$

where in the last equation the square bracket denotes antisymmetrization of the uncontracted indices.

\subsection{The symplectic covariant derivative}

The advantage of the stringy coordinates is that the variable $\Upsilon$ does not enter into symplectic transformations. Thus given any symplectic function $G(\mathcal{Y}, \Upsilon$ ) (not necessarily holomorphic), then the symplectic transformation behaviour is not modified when taking partial derivatives with respect to $\Upsilon$. In particular, if $G(\mathcal{Y}, \Upsilon)$ is a symplectic function, then so is $\partial G / \partial \Upsilon$. In contrast, when using the supergravity variables $Y^{I}$, then partial derivatives with respect to $\Upsilon$ change the symplectic transformation behaviour. For example, while the derivative $F_{\Upsilon}$ of the generalized prepotential is a symplectic function, its derivatives like $F_{\Upsilon \Upsilon}$ are not [9]. Using (3.9) there is a systematic way to compensate for this behaviour. Suppose $G(Y, \Upsilon)$ is a symplectic function, given in supergravity variables, which for the time being we assume to be holomorphic. ${ }^{7}$ Expressing $G$ in stringy variables, we obtain $G=G(Y(\mathcal{Y}, \Upsilon), \Upsilon) .{ }^{8}$ When regarding $G$ as a function of $\mathcal{Y}$ and $\Upsilon$, the partial derivative with respect to $\Upsilon$ is again a symplectic function. Now apply the chain rule:

$$
\left.\frac{\partial G}{\partial \Upsilon}\right|_{\mathcal{Y}}=\left.\frac{\partial G}{\partial \Upsilon}\right|_{Y}+\left.\frac{\partial G}{\partial Y^{I}}\right|_{\Upsilon} \frac{\partial Y^{I}}{\partial \Upsilon}
$$

and use (3.9)

$$
\frac{\partial Y^{I}}{\partial \Upsilon}=-\frac{\partial \Delta Y^{I}}{\partial \Upsilon}=+i N^{I J} F_{J \Upsilon}
$$

Therefore, if $G(Y, \Upsilon)$ is a symplectic function, then

$$
D \Upsilon G:=\left.\frac{\partial G}{\partial \Upsilon}\right|_{Y}+i N^{I J} F_{J \Upsilon} \frac{\partial G}{\partial Y^{I}}
$$

is also symplectic. The expression

$$
D_{\Upsilon}=\left.\frac{\partial}{\partial \Upsilon}\right|_{Y}+i N^{I J} F_{J \Upsilon} \frac{\partial}{\partial Y^{I}},
$$

\footnotetext{
${ }^{7}$ This restriction will be lifted later.

${ }^{8}$ Note that, though we do not indicate this by notation, $Y(\mathcal{Y}, \Upsilon)$ is not holomorphic.
} 
which we have derived from the coordinate transformation between supergravity and stringy variables, is the symplectic covariant derivative which was introduced in [9] based on studying the symplectic transformation behaviour of derivatives of symplectic functions. We remark that while $G$ was assumed holomorphic, $D_{\Upsilon} G$ is not holomorphic, due to the presence of the inverse metric $N^{I J}$. By taking higher covariant derivatives $D_{\Upsilon}^{n} G$, one can create a whole tower of symplectic functions. We remark that when the initial function $G$ is non-holomorphic, the covariant derivative needs to be modified, as will be discussed later.

The main application of this result is to show how one can obtain, starting from $F_{\Upsilon}(Y, \Upsilon)$ a hierarchy of functions $F^{(n)}(\mathcal{Y}, \overline{\mathcal{Y}})$ which can be interpreted as topological free energies, because they satisfy the holomorphic anomaly equation. While this is result known from [9] we briefly explain how this works and how the hierarchy of equations for the functions $F^{(n)}(\mathcal{Y}, \overline{\mathcal{Y}})$ can be consolidated into a master anomaly equation.

First, following [9] we define ${ }^{9}$ a hierarchy of symplectic functions through covariant derivatives of the holomorphic symplectic function $F_{\Upsilon}(Y, \Upsilon)$ :

$$
\Phi^{(n)}(Y, \bar{Y}, \Upsilon, \bar{\Upsilon})=\frac{1}{n !} D_{\Upsilon}^{n-1} F_{\Upsilon}
$$

for $n=1,2, \ldots$, and $\Phi^{(0)}=0$. Then

$$
\begin{aligned}
\Phi^{(1)} & =F_{\Upsilon} \\
\Phi^{(2)} & =\frac{1}{2} D_{\Upsilon} F_{\Upsilon},
\end{aligned}
$$

etc. $\Phi^{(1)}$ is the only holomorphic function in this hierarchy. One computes

$$
\frac{\partial \Phi^{(2)}}{\partial \bar{Y}^{I}}=\frac{i}{2} \frac{\partial N^{J K}}{\partial \bar{Y}^{I}} F_{J \Upsilon} F_{K \Upsilon}=\frac{1}{2} \bar{F}_{I}^{J K} \partial_{J} \Phi^{(1)} \partial_{K} \Phi^{(1)},
$$

where $\bar{F}_{I}^{J K}=\bar{F}_{I P Q} N^{P J} N^{Q K}$. From this starting point it is straightforward to obtain the holomorphic anomaly equation

$$
\frac{\partial \Phi^{(n)}}{\partial \bar{Y}^{I}}=\frac{1}{2} \bar{F}_{I}^{J K} \sum_{r=1}^{n-1} \partial_{J} \Phi^{(r)} \partial_{K} \Phi^{(n-r)}, \quad n \geq 2
$$

by complete induction. Next we define

$$
F^{(n)}(\mathcal{Y}, \overline{\mathcal{Y}})=\Phi^{(n)}(Y, \bar{Y}, \Upsilon=\bar{\Upsilon}=0)
$$

where we used that $Y^{I}=\mathcal{Y}^{I}$ for $\Upsilon=0$. Explicit expressions for $F^{(1)}, F^{(2)}$ and $F^{(3)}$ are given in (C.3) (the normalization used there differs by a factor $2 i$ ).

Setting $\Upsilon=0$, one obtains a holomorphic anomaly equation

$$
\frac{\partial F^{(n)}}{\partial \overline{\mathcal{Y}}^{I}}=\frac{1}{2} \bar{F}_{I(0)}^{J K} \sum_{r=1}^{n-1} \partial_{J} F^{(r)} \partial_{K} F^{(n-r)}, \quad n \geq 2
$$

\footnotetext{
${ }^{9}$ Note that here we use a different normalisation from the one used in section 1.
} 
for the functions $F^{(n)}(\mathcal{Y}, \overline{\mathcal{Y}})$. This is not the full anomaly equation for the genus $n$ topological free energies of the topological string. The reason is that, so far, $F_{\Upsilon}$ and hence $\Phi^{(1)}$ and $F^{(1)}$ are holomorphic, while for the topological string they are not. This will be addressed in the next step where we extend our formalism to the case of a non-holomorphic $F_{\Upsilon}$. For terminological convenience we will refer to the functions $F^{(n)}$ as genus $n$ topological free energies, or free energies for short. ${ }^{10}$

The hierarachy of equations (3.13) can be re-organised into a master anomaly equation for the topological free energy

$$
F_{\text {top }}(\mathcal{Y}, \overline{\mathcal{Y}}, Q)=\sum_{n=1}^{\infty} Q^{n} F^{(n)}(\mathcal{Y}, \overline{\mathcal{Y}}),
$$

where the expansion parameter $Q$ is, for the topological string, related to the topological string coupling. Taking into account that $F^{(1)}$ is (so far) holomorphic, it is straightforward to verify that (3.13) follows from

$$
\frac{\partial F_{\text {top }}}{\partial \overline{\mathcal{Y}}^{I}}=\frac{1}{2} \bar{F}_{I(0)}^{J K} \partial_{J} F_{\text {top }} \partial_{K} F_{\text {top }}
$$

by expansion in $Q$.

Since $F_{\Upsilon}$ is the natural master function in the supergravity formalism, one would like to have a master anomaly equation for it. This is not straightforward, since the Taylor coefficients of $F_{\Upsilon}(Y, \Upsilon)$ with respect to $\Upsilon$ are not symplectic functions. We proceed by expressing $F_{\Upsilon}$ in stringy variables and introducing a shift in $\Upsilon$ :

$$
F_{\Upsilon}(\mathcal{Y}, \overline{\mathcal{Y}}, \Upsilon, \bar{\Upsilon}, Q):=F_{\Upsilon}(\mathcal{Y}, \overline{\mathcal{Y}}, \Upsilon+Q, \bar{\Upsilon})
$$

Then we make a Taylor expansion with respect to the 'fluctuation' $Q$. As indicated by notation, we need to treat $\Upsilon$ and $\bar{\Upsilon}$ as independent variables, and the 'shifted' $F_{\Upsilon}$ is not any more holomorphic in supergravity variables. Also note that when using supergravity variables the dependence on $Q$ is not any more of the form $\Upsilon+Q$.

Now we express the expansion coefficients in supergravity variables:

$$
\begin{aligned}
F_{\Upsilon}(\mathcal{Y}, \overline{\mathcal{Y}}, \Upsilon, \bar{\Upsilon}, Q) & =\sum_{n=1}^{\infty} \frac{1}{n !} Q^{n} \partial_{\Upsilon}^{n} F_{\Upsilon}(\mathcal{Y}, \overline{\mathcal{Y}}, \Upsilon, \bar{\Upsilon})=\sum_{n=1}^{\infty} \frac{1}{n !} Q^{n} D_{\Upsilon}^{n} F_{\Upsilon}(Y, \Upsilon) \\
& =\sum_{n=1}^{\infty} \frac{Q^{n}}{n !}(n+1) ! \Phi^{(n)}(Y, \bar{Y}, \Upsilon, \bar{\Upsilon}) \\
& =\sum_{n=1}^{\infty}(n+1) Q^{n} \Phi^{(n)}(Y, \bar{Y}, \Upsilon, \bar{\Upsilon})
\end{aligned}
$$

Next we integrate with respect to $Q$ :

$$
G(Y, \bar{Y}, \Upsilon, \bar{\Upsilon}, Q)=\sum_{n=0}^{\infty} Q^{n+1} \Phi^{(n+1)}(Y, \bar{Y}, \Upsilon, \bar{\Upsilon})
$$

\footnotetext{
${ }^{10}$ We remark that our formalism is independent of an explicit realization by a concrete topological string model, and in this sense independent of the topological string. Our formalism is a general framework, for which the topological string is one (important) application.
} 
and by setting $\Upsilon=0=\bar{\Upsilon}$ we obtain the topological free energy $F_{\text {top }}$.

$$
\begin{aligned}
G(Y, \bar{Y}, \Upsilon=\bar{\Upsilon}=0, Q) & =G(\mathcal{Y}, \overline{\mathcal{Y}}, \Upsilon=\bar{\Upsilon}=0, Q)=\sum_{n=0}^{\infty} Q^{n+1} F^{(n+1)}(\mathcal{Y}, \overline{\mathcal{Y}}) \\
& =F_{\text {top }}(\mathcal{Y}, \overline{\mathcal{Y}}, Q)
\end{aligned}
$$

The function $G$ satisfies the master anomaly equation

$$
\frac{\partial}{\partial \bar{Y}^{I}} G=\frac{1}{2} \bar{F}_{I}^{J K} \partial_{J} G \partial_{K} G,
$$

which for $\Upsilon=\bar{\Upsilon}=0$ becomes the master anomaly equation (3.14) for the topological free energy.

We note that the relation between the topological free energies $F^{(g)}$ and the function $F_{\Upsilon}$ is complicated. This is of course to be expected from [12]. The reason is that in a Taylor expansion of $F_{\Upsilon}(Y, \Upsilon)$ the coefficients are not symplectic functions. The topological free energies can be regarded as coefficients in a symplectically covariant Taylor expansion, which in practice we cannot manage in closed form but only by evaluating derivatives at $\Upsilon=0$. We also remark that the use of two complementary set of complex coordinates, reflects that there is a tension between holomorphicity and symplecticity. In the supergravity variables we have manifest holomorphicity, but only the full symplectic vector $\left(Y^{I}, F_{I}\right)$ and the full function $F_{\Upsilon}$ are symplectically covariant. If one wants to organise data in a hierarchy of symplectic function, holomorphicity is violated, albeit in a systematic way controlled by the anomaly equation. One can then either work with the covariant derivatives $\Phi^{(n+1)} \simeq D_{\Upsilon}^{n} F_{\Upsilon}$ or use the stringy variables and work with the free energies $F^{(n)}$.

Above we obtained a master equation for $F_{\Upsilon}$ from the master anomaly equation for $F_{\text {top }}$. The result is not quite satisfactory, as we need the background shift $Q$ as a device. But in the next section we will see that a master equation for $F_{\Upsilon}$ can be obtained directly within the supergravity formalism.

\subsection{Anomaly equation from the Hessian structure}

We will now show that the holomorphic anomaly equation arises as an integrability condition for the existence of a Hesse potential on $\hat{M}$. The metric $g^{H}$ being Hessian means that $S=\nabla g^{H}$ is a completely symmetric rank three tensor. In $\nabla$-affine coordinates $Q^{a}=\left(x^{I}, y_{I}, \Upsilon, \bar{\Upsilon}\right)$ the components of the tensor $S$ are simply the third partial derivatives of the Hesse potential, or equivalently the first partial derivatives of the metric, and therefore proportional to the Christoffel symbols of the first kind (which for a Hessian metric are completely symmetric with respect to $\nabla$-affine coordinates):

$$
S_{a b c}=\partial_{a b c}^{3} H=\partial_{a} g_{b c}^{H} .
$$

One particular relation is

$$
S_{x^{I} \Upsilon \Upsilon}=S_{\Upsilon x^{I} \Upsilon}
$$

or

$$
\partial_{x^{I}} g_{\Upsilon \Upsilon}^{H}=\partial_{\Upsilon} g_{x^{I} \Upsilon}^{H}
$$


where

$$
g_{\Upsilon \Upsilon}^{H}=-i D_{\Upsilon} F_{\Upsilon}
$$

and

$$
g_{x^{I} \Upsilon}^{H}=2 \bar{F}_{I J} N^{J K} F_{K \Upsilon} .
$$

We now evaluate equation (3.15) in supergravity coordinates $\left(Y^{I}, \bar{Y}^{I}, \Upsilon, \bar{\Upsilon}\right)$, using the corresponding Jacobian to obtain

$$
S_{x^{I} \Upsilon \Upsilon}=\left.\frac{\partial g_{\Upsilon \Upsilon}^{H}}{\partial x^{I}}\right|_{y}=\left.\frac{\partial g_{\Upsilon \Upsilon}^{H}}{\partial x^{I}}\right|_{u}+\frac{\partial u^{K}}{\partial x^{I}} \frac{\partial g_{\Upsilon \Upsilon}^{H}}{\partial u^{K}}, \text { where }\left.\quad \frac{\partial}{\partial x^{I}}\right|_{u}=\frac{\partial}{\partial Y^{I}}+\frac{\partial}{\partial \bar{Y}^{I}}
$$

and

$$
S_{\Upsilon x^{I} \Upsilon}=\left.\frac{\partial g_{x^{I} \Upsilon}^{H}}{\partial \Upsilon}\right|_{x, y}=\left.\frac{\partial g_{x^{I} \Upsilon}^{H}}{\partial \Upsilon}\right|_{x, u}+\frac{\partial u^{K}}{\partial \Upsilon} \frac{\partial g_{x^{I} \Upsilon}^{H}}{\partial u^{K}} .
$$

We find for the various terms,

$$
\begin{aligned}
\left.\frac{\partial g_{\Upsilon \Upsilon}^{H}}{\partial x^{I}}\right|_{u}= & -i \frac{\partial}{\partial \bar{Y}^{I}} D_{\Upsilon} F_{\Upsilon}-i \bar{F}_{\bar{I}}^{K L} F_{K \Upsilon} F_{L \Upsilon}, \\
\left.\frac{\partial g_{\Upsilon \Upsilon}^{H}}{\partial u^{K}}\right|_{x}= & \left(\frac{\partial}{\partial Y^{K}}-\frac{\partial}{\partial \bar{Y}^{K}}\right)\left(F_{\Upsilon \Upsilon}+i N^{K L} F_{K \Upsilon} F_{L \Upsilon}\right) \\
= & F_{K \Upsilon \Upsilon}-F_{K}^{P Q} F_{P \Upsilon} F_{Q \Upsilon}-2 N^{P Q} F_{K P \Upsilon} F_{Q \Upsilon}-\bar{F}_{K}{ }^{P Q} F_{P \Upsilon} F_{Q \Upsilon}, \\
\left.\frac{\partial g_{x^{I} \Upsilon}^{H}}{\partial \Upsilon}\right|_{x, u}= & -i F_{I \Upsilon \Upsilon}+F_{\Upsilon I}^{J} F_{J \Upsilon}+\left(F_{I J}+\bar{F}_{I J}\right)\left(i F_{\Upsilon}^{J K} F_{K \Upsilon}+F_{\Upsilon \Upsilon}^{J}\right), \\
\left.\frac{\partial g_{x^{I} \Upsilon}^{H}}{\partial u^{K}}\right|_{x}= & F_{I K \Upsilon}+i F_{I K}{ }^{L} F_{L \Upsilon}+i\left(F_{I L}+\bar{F}_{I L}\right)\left(i F_{K}{ }^{L P} F_{P \Upsilon}+F_{\Upsilon K}{ }^{L}\right) \\
& -i \bar{F}_{I K}{ }^{L} F_{L \Upsilon}-\left(F_{I L}+\bar{F}_{I L}\right) \bar{F}_{K}{ }^{L P} F_{P \Upsilon},
\end{aligned}
$$

where indices are raised using $N^{I J}$. Then, the Hessian condition (3.15) results in

$$
\frac{\partial}{\partial \bar{Y}^{I}} D_{\Upsilon} F_{\Upsilon}=\bar{F}_{I J K} N^{J P} N^{K Q} F_{P \Upsilon} F_{Q \Upsilon}
$$

In the holomorphic case at hand $\left(\partial_{\bar{I}} F_{\Upsilon}=0\right)$, this equation can be regarded as a master anomaly equation in supergravity variables. First note that for $\Upsilon=0$ (3.17) reduces to the anomaly equation for $F^{(2)}$. The anomaly equations for $F^{(n)}$ with $n>2$ are obtained by covariant differentiation of (3.17). Here one uses that holomorphicity of the generalized prepotential implies

$$
\partial_{\bar{I}} F_{\Upsilon}=0, \quad D_{\Upsilon} \bar{F}_{I J K}=0
$$

and one also uses the identity [13]

$$
\left[D_{\Upsilon}, N^{I J} \partial_{J}\right]=0
$$


For example, to derive the anomaly equation for $\Phi^{(3)}$ and, hence, for $F^{(3)}$ we need to evaluate

$$
\begin{aligned}
\partial_{\bar{I}} D_{\Upsilon}^{2} F_{\Upsilon} & =D_{\Upsilon} \partial_{\bar{I}} D_{\Upsilon} F_{\Upsilon}+i\left(\partial_{\bar{I}} N^{J K}\right) F_{J \Upsilon} \partial_{K} D_{\Upsilon} F_{\Upsilon} \\
& =3 \bar{F}_{I}^{J K} \partial_{J} F_{\Upsilon} \partial_{K} D_{\Upsilon} F_{\Upsilon} .
\end{aligned}
$$

Using that $D_{\Upsilon}^{n-1} F_{\Upsilon}=n ! \Phi^{(n)}$ this becomes

$$
\partial_{\bar{I}} \Phi^{(3)}=\bar{F}_{I}^{J K} \partial_{J} \Phi^{(1)} \partial_{K} \Phi^{(2)}=\frac{1}{2} \bar{F}_{I}^{J K} \sum_{r=1}^{2} \partial_{J} \Phi^{(r)} \partial_{K} \Phi^{(3-r)}
$$

which for $\Upsilon=0$ is the anomaly equation for $F^{(3)}$. Proceeding by induction one obtains the full hierarchy (3.11) of anomaly equations.

One may ask whether other components of $S$ will give rise to additional non-trivial differential equations. To investigate this, we now consider the component $S_{x^{I} \Upsilon \bar{\Upsilon}}=\partial_{x^{I}} g_{\Upsilon \bar{\Upsilon}}^{H}$, which is constructed out of the metric component $g_{\Upsilon \bar{\Upsilon}}^{H}=N^{I J} F_{I \Upsilon} \bar{F}_{J \Upsilon}$. Evaluating the relation $S_{x^{I} \Upsilon \bar{\Upsilon}}=S_{\bar{\Upsilon} x^{I} \Upsilon}=\partial_{\bar{\Upsilon}} g_{x^{I} \Upsilon}^{H}$ in supergravity variables we find that it is identically satisfied. Thus, the only non-trivial differential equation resulting from $g_{\Upsilon \Upsilon}^{H}$ and $g_{\Upsilon \bar{\Upsilon}}^{H}$ is encoded in the relation $S_{x^{I} \Upsilon \Upsilon}=S_{\Upsilon x^{I} \Upsilon}$.

\section{The non-holomorphic deformation}

So far we have assumed that $F(Y, \Upsilon)$ and, hence, $F_{\Upsilon}$ are holomorphic in the supergravity variables, which implies that $F^{(1)}(\mathcal{Y})$ is also holomorphic, while $F^{(g)}(\mathcal{Y}, \overline{\mathcal{Y}})$ with $g>1$ satisfy the anomaly equation (3.13). For the topological string the situation is more complicated since already $F^{(1)}(\mathcal{Y}, \overline{\mathcal{Y}})$ is non-holomorphic. We therefore now generalize our framework and induce geometric data on $\hat{M}$ using a non-holomorphic map $\phi: \hat{M} \rightarrow V$, which then corresponds to a non-holomorphic generalized prepotential $F=F(Y, \bar{Y}, \Upsilon, \bar{\Upsilon})$. This explicit non-holomorphicity will in turn modify the anomaly equation (3.13) satisfied by the topological free energies $F^{(g)}(\mathcal{Y}, \overline{\mathcal{Y}})$. The precise form of the modification depends on the details of the non-holomorphic deformation. We will first keep the discussion general, and later show that when chosing a particular non-holomorphic deformation we obtain the correct full anomaly equation (at least to leading order in a formal expansion we will explain later). As discussed in [13] there are other types of non-holomorphic deformations that are, for example, relevant for non-linear deformations of electrodynamics. Any such deformation could be analyzed in the framework of our formalism.

Since $F$ and $F_{\Upsilon}$ are no longer holomorphic, they will have non-vanishing derivatives with respect to $\bar{Y}^{I}$ and $\bar{\Upsilon}$. In the following we will use a notation involving 'unbarred' indices $I, J, \ldots$ and 'barred' indices $\bar{I}, \bar{J}, \ldots$.

\subsection{Non-holomorphic deformation of the prepotential}

We generalize the map (3.1) to

$$
\phi: \hat{M}=M \times \mathbb{C} \rightarrow V, \quad\left(Y^{I}, \Upsilon\right) \mapsto\left(Y^{I}, F_{I}(Y, \bar{Y}, \Upsilon, \bar{\Upsilon})\right),
$$


where $F_{I}=\partial F / \partial Y^{I}$, can be obtained from a generalized prepotential $F$. We assume that $F$ has the form [18]

$$
F(Y, \bar{Y}, \Upsilon, \bar{\Upsilon})=F^{(0)}(Y)+2 i \Omega(Y, \bar{Y}, \Upsilon, \bar{\Upsilon})
$$

where $F^{(0)}$ is the undeformed prepotential, and where $\Omega$ is real-valued. ${ }^{11}$ The holomorphic deformation is recovered when $\Omega$ is harmonic. This makes use of the observation that the complex symplectic vector $\left(Y^{I}, F_{I}\right)$ does not uniquely determine the prepotential $F$ [12]. If we make a transformation

$$
\begin{aligned}
F^{(0)}(Y) & \rightarrow F^{(0)}(Y)+g(Y, \Upsilon) \\
\Omega(Y, \bar{Y}, \Upsilon, \bar{\Upsilon}) & \rightarrow \Omega(Y, \bar{Y}, \Upsilon, \bar{\Upsilon})-\frac{1}{2 i}(g(Y, \Upsilon)-\bar{g}(\bar{Y}, \bar{\Upsilon}))
\end{aligned}
$$

where $g(Y, \Upsilon)$ is holomorphic, then $F$ changes by an antiholomorphic function, $F \rightarrow F+\bar{g}$, and the symplectic vector $\left(Y^{I}, F_{I}\right)$ and the map $\phi$ are invariant. If $\Omega$ is harmonic,

$$
\Omega(Y, \bar{Y}, \Upsilon, \bar{\Upsilon})=f(Y, \Upsilon)+\bar{f}(\bar{Y}, \bar{\Upsilon})
$$

we can make a transformation with $g=2 i f$ and obtain

$$
F \rightarrow F^{(0)}(Y)+2 i f(Y, \Upsilon)=: F(Y, \Upsilon)
$$

which is a holomorphically deformed prepotential, as considered in the previous section. If, however, $\Omega$ is not harmonic, then we have a genuine generalization which requires us to consider non-holomorphic generalized prepotentials. For the case of the topological string, it is convenient to split the non-holomorphic generalized prepotential as in (4.2) into the undeformed prepotential $F^{(0)}$ and a real-valued non-harmonic function $\Omega$ which encodes all higher derivative effects, holomorphic as well as non-holomorphic.

We proceed by analysing the geometry induced by pulling back the standard hermitian form $\gamma_{V}$ of $V$ given by (2.3) to $\hat{M}$ using (4.1):

$$
\begin{aligned}
\gamma= & -i\left(F_{I J}^{(0)}-\bar{F}_{\bar{I} \bar{J}}^{(0)}\right) d Y^{I} \otimes d \bar{Y}^{J}+2\left(\Omega_{I J}+\Omega_{\bar{I} \bar{J}}\right) d Y^{I} \otimes d \bar{Y}^{J}+2 \Omega_{\bar{I} J} d Y^{I} \otimes d Y^{J} \\
& +2 \Omega_{I \bar{J}} d \bar{Y}^{I} \otimes d \bar{Y}^{J}+2 \Omega_{\bar{I} \bar{\Upsilon}} d Y^{I} \otimes d \bar{\Upsilon}+2 \Omega_{I \Upsilon} d \Upsilon \otimes d \bar{Y}^{I}+2 \Omega_{\bar{I} \Upsilon} d Y^{I} \otimes d \Upsilon \\
& +2 \Omega_{I \bar{\Upsilon}} d \bar{\Upsilon} \otimes d \bar{Y}^{I}
\end{aligned}
$$

By decomposing $\gamma=g+i \omega$, we obtain the following metric on $\hat{M}$ :

$$
\begin{aligned}
g= & -i\left(F_{I J}^{(0)}-\bar{F}_{\bar{I} \bar{J}}^{(0)}\right) d Y^{I} d \bar{Y}^{J}+2\left(\Omega_{I J}+\Omega_{\bar{I} \bar{J}}\right) d Y^{I} d \bar{Y}^{J}+2 \Omega_{\bar{I} J} d Y^{I} d Y^{J}+2 \Omega_{I \bar{J}} d \bar{Y}^{I} d \bar{Y}^{J} \\
& +2 \Omega_{\bar{I} \bar{\Upsilon}} d Y^{I} d \bar{\Upsilon}+2 \Omega_{I \Upsilon} d \Upsilon d \bar{Y}^{I}+2 \Omega_{\bar{I} \Upsilon} d Y^{I} d \Upsilon+2 \Omega_{I \bar{\Upsilon}} d \bar{\Upsilon} d \bar{Y}^{I} .
\end{aligned}
$$

From this expression it is manifest that $g$ is not Hermitian, and hence not Kähler with respect to the natural complex structure $J$. The non-Hermiticity is encoded in the mixed

\footnotetext{
${ }^{11}$ This function is not to be confused with the complex symplectic form on the vector space $V$ introduced in subsection 2.1 .
} 
derivatives $\Omega_{I \bar{J}}$, which makes it manifest that it is related to the non-harmonicity of $\Omega$. This metric occurs in the sigma model discussed in [17].

The imaginary part of $\gamma$ defines a two-form on $\hat{M}$ :

$$
\begin{aligned}
\omega= & \frac{1}{2 i}\left(-i\left(F_{I J}^{(0)}-\bar{F}_{\bar{I} \bar{J}}^{(0)}\right)\right) d Y^{I} \wedge d \bar{Y}^{J}-i\left(\Omega_{\bar{I} \bar{J}}+\Omega_{I J}\right) d Y^{I} \wedge d \bar{Y}^{J} \\
& -i \Omega_{\bar{I} J} d Y^{I} \wedge d Y^{J}+i \Omega_{I \bar{J}} d \bar{Y}^{I} \wedge d \bar{Y}^{J}-i \Omega_{\bar{I} \bar{\Upsilon}} d Y^{I} \wedge d \bar{\Upsilon}-i \Omega_{I \Upsilon} d \Upsilon \wedge d \bar{Y}^{I} \\
& -i \Omega_{\bar{I} \Upsilon} d Y^{I} \wedge d \Upsilon+i \Omega_{I \bar{\Upsilon}} d \bar{Y}^{I} \wedge d \bar{\Upsilon} .
\end{aligned}
$$

This two-form is no longer of type $(1,1)$ with respect to the standard complex structure, which is consistent with the non-Hermiticity of $g$. However, $\omega$ is still closed

$$
d \omega=0,
$$

so that $(\hat{M}, \omega)$ is at least a symplectic manifold.

Comparing $\omega$ to $d x^{I} \wedge d y^{I}$, we find:

$$
\begin{aligned}
2 d x^{I} \wedge d y_{I}= & \omega+2 i \Omega_{I \bar{J}} d Y^{I} \wedge d \bar{Y}^{J}+i \Omega_{I \Upsilon} d Y^{I} \wedge d \Upsilon-i \Omega_{\bar{I} \bar{\Upsilon}} d \bar{Y}^{I} \wedge d \bar{\Upsilon} \\
& +i \Omega_{I \bar{\Upsilon}} d Y^{I} \wedge d \bar{\Upsilon}-i \Omega_{\bar{I} \bar{\Upsilon}} d \bar{Y}^{I} \wedge d \Upsilon .
\end{aligned}
$$

As a consistency check, we verify that $2 d x^{I} \wedge d y_{I}-\omega$ is closed:

$$
2 d x^{I} \wedge d y_{I}=\omega-\frac{1}{2} d\left(\Upsilon d F_{\Upsilon}\right)-\frac{1}{2} d\left(\bar{\Upsilon} d F_{\bar{\Upsilon}}\right)-\partial \bar{\partial} F,
$$

where $\partial=d Y^{I} \otimes \partial_{Y^{I}}+d \Upsilon \otimes \partial_{\Upsilon}$. Note that the difference between the symplectic form $2 d x^{I} \wedge d y_{I}$ of $M$ and the symplectic form $\omega$ of $\hat{M}$ is still exact. Compared to (3.4) we have an additional term which measures the non-holomorphicity of the generalized prepotential.

\subsection{Real coordinates and the Hesse potential}

To convert from complex supergravity variables we need to generalize our previous calculation of the Jacobian and its inverse:

$$
\frac{D(x, y, \Upsilon, \bar{\Upsilon})}{D(x, u, \Upsilon, \bar{\Upsilon})}=\left(\begin{array}{cccc}
\mathbb{1} & 0 & 0 & 0 \\
\frac{1}{2} R_{+} & -\frac{1}{2} N_{-} & \frac{1}{2}\left(F_{I \Upsilon}+\bar{F}_{\bar{I} \Upsilon}\right) & \frac{1}{2}\left(\bar{F}_{\bar{I} \bar{\Upsilon}}+F_{I \bar{\Upsilon}}\right) \\
0 & 0 & \mathbb{1} & 0 \\
0 & 0 & 0 & \mathbb{1}
\end{array}\right)
$$

and, by a straigtforward matrix inversion

$$
\frac{D(x, u, \Upsilon, \bar{\Upsilon})}{D(x, y, \Upsilon, \bar{\Upsilon})}=\left(\begin{array}{cccc}
\mathbb{1} & 0 & 0 & 0 \\
N_{-}^{-1} R_{+} & -2 N_{-}^{-1} & N_{-}^{-1}\left(F_{I \Upsilon}+\bar{F}_{\bar{I} \Upsilon}\right) & N_{-}^{-1}\left(\bar{F}_{\bar{I} \bar{\Upsilon}}+F_{I \bar{\Upsilon}}\right) \\
0 & 0 & \mathbb{1} & 0 \\
0 & 0 & 0 & \mathbb{1}
\end{array}\right)
$$

This reduces to the previous result when switching off the non-holomorphic deformation. When restricting to the left upper block, the result agrees with [17]. We have used the following definitions [17]:

$$
N_{ \pm I J}=N_{I J} \pm 2 \operatorname{Im} F_{I \bar{J}}=-i\left(F_{I J}-\bar{F}_{\bar{I} \bar{J}} \pm F_{I \bar{J}} \mp \bar{F}_{\bar{I} J}\right)
$$


and

$$
R_{ \pm I J}=R_{I J} \pm 2 \operatorname{Re} F_{I \bar{J}}=F_{I J}+\bar{F}_{\bar{I} \bar{J}} \pm F_{I \bar{J}} \pm \bar{F}_{\bar{I} J} .
$$

Note that $N_{-}^{T}=N_{-}$, while $R_{ \pm}^{T}=R_{\mp}$.

As already observed in [18], in the presence of explicit non-holomorphic deformations the Hesse potential is not to be defined as the Legendre transform of $2 \operatorname{Im} F$ but rather as the Legendre of

$$
L=2 \operatorname{Im} F-2 \Omega=2 \operatorname{Im} F^{(0)}+2 \Omega .
$$

As explained in $[12,13]$, the function $L$ can be interpreted as a Lagrange function, and the Hesse potential as the corresponding Hamilton function. ${ }^{12}$ Thus the Hesse potential associated to a non-holomorphically deformed prepotential is

$$
H(x, y, \Upsilon, \bar{\Upsilon})=-i(F-\bar{F})-2 \Omega-2 u^{I} y_{I}
$$

By taking derivatives with respect to the real coordinates $\left(Q^{A}\right)=\left(q^{a}, \Upsilon, \bar{\Upsilon}\right)$, where $\left(q^{a}\right)=$ $\left(x^{I}, y_{I}\right)$ we obtain the components of a Hessian metric:

$$
\begin{aligned}
\frac{\partial H}{\partial q^{a} \partial q^{b}} & =\left(\begin{array}{cc}
N_{+}+R_{-} N_{-}^{-1} R_{+} & -2 R_{-} N_{-}^{-1} \\
-2 N_{-}^{-1} R_{+} & 4 N_{-}^{-1}
\end{array}\right), \\
\frac{\partial^{2} H}{\partial x^{I} \partial \Upsilon} & =-i\left(F_{I \Upsilon}-\bar{F}_{\bar{I} \Upsilon}\right)+R_{-I K} N_{-}^{K J}\left(F_{J \Upsilon}+\bar{F}_{\bar{J} \Upsilon}\right), \\
\frac{\partial^{2} H}{\partial y_{I} \partial \Upsilon} & =-2 N_{-}^{I K}\left(F_{K \Upsilon}+\bar{F}_{\bar{K} \Upsilon}\right),
\end{aligned}
$$

together with their complex conjugates and

$$
\begin{aligned}
& \frac{\partial^{2} H}{\partial \Upsilon \partial \bar{\Upsilon}}=-i F_{\Upsilon \bar{\Upsilon}}+N_{-}^{I J}\left(\bar{F}_{\bar{I} \bar{\Upsilon}}-\bar{F}_{I \bar{\Upsilon}}\right)\left(F_{\Upsilon J}-F_{\Upsilon \bar{J}}\right)=-i D_{\Upsilon} F_{\bar{\Upsilon}}, \\
& \frac{\partial^{2} H}{\partial \Upsilon \partial \Upsilon}=-i D_{\Upsilon} F_{\Upsilon}, \quad \frac{\partial^{2} H}{\partial \bar{\Upsilon} \partial \bar{\Upsilon}}=i \overline{D_{\Upsilon} F_{\Upsilon}},
\end{aligned}
$$

where

$$
D_{\Upsilon}=\partial \Upsilon+i N_{-}^{I J}\left(F_{\Upsilon J}-F_{\Upsilon \bar{J}}\right)\left(\frac{\partial}{\partial Y^{I}}-\frac{\partial}{\partial \bar{Y}^{I}}\right)
$$

is the symplectic covariant derivative introduced in [13]. This covariant derivative is a generalization of (3.10) which generates a hierarchy of symplectic functions starting from a non-holomorphic symplectic function. For holomorphic symplectic functions it reduces to (3.10). We will show below that $D_{\Upsilon}$ can be derived by transforming the partial derivative $\left.\partial_{\Upsilon}\right|_{\mathcal{Y}}$ from stringy variables to supergravity variables.

As before, the Hessian metric $g^{H}$ differs from the metric $g$ induced by pulling back $g_{V}$ using $\phi$ by differentials involving derivatives of $H$ with respect to $\Upsilon, \bar{\Upsilon}$ :

$$
g^{H}=g+\left.\partial^{2} H\right|_{x, y}
$$

where

$$
\left.\partial^{2} H\right|_{x, y}=\frac{\partial^{2} H}{\partial \Upsilon \partial \Upsilon} d \Upsilon d \Upsilon+2 \frac{\partial^{2} H}{\partial \Upsilon \partial \bar{\Upsilon}} d \Upsilon d \bar{\Upsilon}+\frac{\partial^{2} H}{\partial \bar{\Upsilon} \partial \bar{\Upsilon}} d \bar{\Upsilon} d \bar{\Upsilon}
$$

\footnotetext{
${ }^{12} \operatorname{In}[12,13]$ the Hesse potential is normalized differently by a factor 2 compared to [18] and the present paper.
} 


\subsection{The symplectic covariant derivative}

As before we can use (4.3) and (4.4) to obtain exact information about the coordinate transformation $\Delta Y^{I}=\mathcal{Y}^{I}-Y^{I}$ between supergravity variables and stringy variables. By proceeding as in subsection 3.4, namely converting the expression (4.4) from supergravity variables to stringy variables, and then converting the result to real variables, we find the following consistency condition:

$$
\frac{\partial \Delta Y^{I}}{\partial \Upsilon}=-i \hat{N}^{I K}\left(F_{K \Upsilon}+\bar{F}_{\bar{K} \Upsilon}\right)
$$

where $\hat{N}^{I J}$ is the inverse of the matrix

$$
\begin{aligned}
\hat{N}_{I J} & =N_{I J}+i F_{J \bar{I}}-i \bar{F}_{\bar{J} I}=-i\left(F_{I J}-\bar{F}_{\bar{I} \bar{J}}-F_{\bar{I} J}+\bar{F}_{I \bar{J}}\right) \\
& =-i\left(F_{I J}-\bar{F}_{\bar{I} \bar{J}}-2 i \Omega_{J \bar{I}}-2 i \Omega_{\bar{J} I}\right)
\end{aligned}
$$

defined in [13]. Note that $\hat{N}_{I J}=N_{-I J}$, which was defined before. The formula (4.7) can be used to derive a modified symplectically covariant derivative, which allows to generate new symplectic functions given a non-holomorphic symplectic function $G(Y, \bar{Y}, \Upsilon, \bar{\Upsilon})$. Indeed, if such a function is given we can express it in stringy variables, $G=G(Y(\mathcal{Y}, \overline{\mathcal{Y}}, \Upsilon, \bar{\Upsilon}), \bar{Y}(\mathcal{Y}, \overline{\mathcal{Y}}, \Upsilon, \bar{\Upsilon}), \Upsilon, \bar{\Upsilon})$, and we know that $\partial G /\left.\partial \Upsilon\right|_{\mathcal{Y}}$ is a symplectic function. Expressing this function in supergravity variables we obtain

$$
\left.\frac{\partial}{\partial \Upsilon}\right|_{\mathcal{Y}} G=\left.\frac{\partial}{\partial \Upsilon}\right|_{Y} G-\frac{\partial \Delta Y^{I}}{\partial \Upsilon}\left(\partial_{Y^{I}}-\partial_{\bar{Y}^{I}}\right) G=: D_{\Upsilon} G
$$

where $D_{\Upsilon}$ denotes the symplectically covariant derivative introduced in (4.6), as can be readily verified by using $F_{\Upsilon}=2 i \Omega_{\Upsilon}$, where $\Omega$ is real valued, which implies $\bar{F}_{\bar{I} \Upsilon}=-F_{\Upsilon \bar{I}}$. This derivative operator was already found in [13] based on studying the symplectic transformation of derivatives of a non-holomorphic generalized prepotential. We have now derived this covariant derivative from a coordinate transformation.

The symplectically covariant derivative $D_{\Upsilon}$ can be applied to any non-holomorphic symplectic function. Thus, we can now construct a hierarchy of symplectic functions starting from a non-holomorphic $F_{\Upsilon}$ :

$$
\Phi^{(n+1)}=\frac{1}{(n+1) !} D_{\Upsilon}^{n} F_{\Upsilon}(Y, \bar{Y}, \Upsilon, \bar{\Upsilon})
$$

As before, we define topological free energies by

$$
F^{(n)}(\mathcal{Y}, \overline{\mathcal{Y}}):=\left.\Phi^{(n)}(Y, \bar{Y}, \Upsilon, \bar{\Upsilon})\right|_{\Upsilon=0}, \quad n \geq 2 .
$$

These functions will satisfy a holomorphic anomaly equation, whose precise form depends on the details of the non-holomorphic deformation. 


\subsection{The holomorphic anomaly equation}

We would like to show that for a suitable choice of a non-holomorphic deformation we obtain the holomorphic anomaly equation of the topological string. As anticipated from [12] this is laborious to do explicitly, since an explicit non-holomorphic deformation leads to a proliferation of non-holomorphic terms. As in [12] we will resort to a (formal) series expansion in parameters which control the non-holomorphicity, and rely on results about the symplectic transformation behaviour of various quantites.

The non-holomorphic dependence of the topological free energies $F^{(g)}$ is entirely encoded in $N_{(0)}^{I J}$ (which, we recall, is the inverse of $N_{I J}^{(0)}=-i\left(F_{I J}^{(0)}-\bar{F}_{I J}^{(0)}\right)$ ). The higher $F^{(g)}$ (with $g \geq 2$ ) are polynomials of degree $3 g-3$ in $N_{(0)}^{I J}$, while $F^{(1)}$ depends on the logarithm of $\operatorname{det} N_{(0)}^{I J}$. In the following, we will focus on the polynomial dependence on $N_{I J}^{(0)}$ of the higher $F^{(g)}$, keeping $F^{(1)}$ holomorphic for the time being. We thus consider the deformation

$$
\Omega(Y, \bar{Y}, \Upsilon, \bar{\Upsilon})=f(Y, \Upsilon)+2 \beta \Upsilon N_{(0)}^{I J} f_{I J}(Y, \Upsilon)+\text { c.c } .
$$

where the departure from harmonicity is encoded in $N_{(0)}^{I J}$. We will work to first order in the deformation parameter $\beta$ and in $N_{(0)}^{I J}$ to avoid a proliferation of new terms compared to the holomorphic case. Note that in applications $\beta$ and $N_{(0)}^{I J}$ are not necessarily small, so that the expansion is formal. The above defines a toy model that, as we will see, reproduces the holomorphic anomaly equation for the topological free energies $F^{(g)}$ for $g \geq 2$, to leading order in $\beta$ and in $N_{(0)}^{I J}$. Since $F^{(1)}$ is still holomorphic, this toy model does not fully capture the topological string. We will address this issue at the end of this section.

Expanding

$$
f(Y, \Upsilon)=\sum_{n=1}^{\infty} \Upsilon^{n} f^{(n)}(Y) \Rightarrow f_{\Upsilon}=\sum_{n=1}^{\infty} n \Upsilon^{n-1} f^{(n)}(Y)
$$

we obtain from (4.9)

$$
\Omega_{\Upsilon}=f^{(1)}+\sum_{n=2}^{\infty} n \Upsilon^{n-1}\left(f^{(n)}(Y)+2 \beta N_{(0)}^{I J} f_{I J}^{(n-1)}\right) .
$$

To first order in $\beta$ and in $N_{(0)}^{I J}$, the function $F_{\Upsilon}=2 i \Omega_{\Upsilon}$, given by

$$
\begin{aligned}
F_{\Upsilon}(Y, \bar{Y}, \Upsilon)= & 2 i\left[f_{\Upsilon}+2 \beta N_{(0)}^{I J} f_{I J}(Y, \Upsilon)+2 \beta \Upsilon N_{(0)}^{I J} f_{\Upsilon I J}(Y, \Upsilon)\right] \\
=2 i\left[f^{(1)}(Y)+2 \Upsilon\right. & \left(f^{(2)}(Y)+2 \beta N_{(0)}^{I J} f_{I J}^{(1)}(Y)\right) \\
& \left.+3 \Upsilon^{2}\left(f^{(3)}(Y)+2 \beta N_{(0)}^{I J} f_{I J}^{(2)}(Y)\right)+\cdots\right],
\end{aligned}
$$

transforms as a function under symplectic transformations provided we modify the transformation behaviour of $f(Y, \Upsilon)$ to (note that we are using supergravity coordinates $Y^{I}$ ),

$$
f(Y, \Upsilon) \longrightarrow f(Y, \Upsilon)+2 i \beta \Upsilon \mathcal{Z}_{0}^{I J} f_{I J}(Y, \Upsilon)
$$


to first order in $\beta$ and in $\mathcal{Z}_{0}^{I J}$. Here, $\mathcal{Z}_{0}^{I J}$ denotes the transformation matrix given in (C.2). Note that $\beta N_{(0)}^{I J} f_{I J}$ transforms as follows [12] under symplectic transformations, to first order in $\beta$ and in $N_{(0)}^{I J}\left(\right.$ or $\left.\mathcal{Z}_{0}^{I J}\right)$,

$$
\begin{aligned}
\beta N_{(0)}^{I J} f_{I J} & \longrightarrow \beta\left(N_{(0)}^{I J}-i \mathcal{Z}_{0}^{I J}\right)\left(f_{I J}-F_{I J L}^{(0)} \mathcal{Z}_{0}^{L P} f_{P}\right) \\
& =\beta\left(N_{(0)}^{I J}-i \mathcal{Z}_{0}^{I J}\right) f_{I J}+\mathcal{O}\left(N^{-1} \mathcal{Z}_{0}, \mathcal{Z}_{0}^{2}\right)
\end{aligned}
$$

Using (4.13) and (4.14), it follows that (4.12) is a symplectic function at this order.

We now observe that

$$
F_{\bar{I} \Upsilon} \propto \beta \partial_{\bar{I}} N_{(0)}^{J K}
$$

which is of higher order in $N_{(0)}^{-1}$, and hence will be dropped. Thus

$$
\left.D_{\Upsilon}^{n} F_{\Upsilon}\right|_{\Upsilon=0}=\left.D_{(0) \Upsilon}^{n} F_{\Upsilon}\right|_{\Upsilon=0}+O\left(\left(N_{(0)}^{-1}\right)^{2}\right)
$$

where by

$$
D_{(0) \Upsilon}=\frac{\partial}{\partial \Upsilon}+i N^{I J} F_{I \Upsilon} \frac{\partial}{\partial Y^{J}}
$$

we denote the symplectically covariant derivative (3.10). Thus, while when starting with a non-holomorphic function (3.10) must normally be replaced by (4.6), we neglect the additional terms in the present context because they will necessarily bring in higher powers of $N_{(0)}^{-1}$.

Hence, by working to order $\beta$ and neglecting terms of order $\left(N_{(0)}^{-1}\right)^{2}$, we obtain

$$
\begin{aligned}
\left.D_{\Upsilon}^{n} F_{\Upsilon}\right|_{\Upsilon=0} & =\left.D_{(0) \Upsilon}^{n}\left(2 i f_{\Upsilon}\right)\right|_{\Upsilon=0}+\left.4 i \beta N_{(0)}^{I J} \partial_{\Upsilon}^{n}\left(\sum_{m=1}^{\infty}(m+1) \Upsilon^{m} f_{I J}^{(m)}\right)\right|_{\Upsilon=0} \\
& =\left.D_{(0) \Upsilon}^{n}\left(2 i f_{\Upsilon}\right)\right|_{\Upsilon=0}+4 i \beta(n+1) ! N_{(0)}^{I J} f_{I J}^{(n)} .
\end{aligned}
$$

Now we consider the hierarchy (4.8),

$$
\begin{aligned}
F^{(n+1)} & =\left.\frac{D_{\Upsilon}^{n} F_{\Upsilon}}{(n+1) !}\right|_{\Upsilon=0} \\
& =\left.\frac{1}{(n+1) !} D_{(0) \Upsilon}^{n}\left(2 i f_{\Upsilon}\right)\right|_{\Upsilon=0}+\left.2 \beta N_{(0)}^{I J}\left(2 i f_{I J}^{(n)}\right)\right|_{\Upsilon=0} r+O\left(\beta^{2},\left(N_{(0)}^{-1}\right)^{2}\right) .
\end{aligned}
$$

For $\beta=0$ only the first term $F_{\text {holo }}^{(n+1)}=\left.[(n+1) !]^{-1} D_{(0) \Upsilon}^{n}\left(2 i f_{\Upsilon}\right)\right|_{\Upsilon=0}$ is present, which satisfies the anomaly equation (3.13). Including the deformation term of order $\beta$ we get (note that $Y^{I}=\mathcal{Y}^{I}$ when $\Upsilon=0$ )

$$
\begin{aligned}
\frac{\partial}{\partial \overline{\mathcal{Y}}^{K}} F^{(n+1)} & =\frac{\partial}{\partial \overline{\mathcal{Y}}^{K}} F_{\text {holo }}^{(n+1)}-\left.2 i \beta \bar{F}_{K}^{(0) I J}\left(2 i f_{I J}^{(n)}\right)\right|_{\Upsilon=0} \\
& =\frac{1}{2} \bar{F}_{K}^{(0) I J}\left(\sum_{r=1}^{n} \partial_{I} F_{\text {holo }}^{(r)} \partial_{J} F_{\text {holo }}^{(n+1-r)}-4 i \beta F_{(\text {holo }) I J}^{(n)}\right)
\end{aligned}
$$


Redefining $F^{(n)} \rightarrow 2 i F^{(n)}$ (with $n \geq 1$ ), we obtain

$$
\frac{\partial}{\partial \overline{\mathcal{Y}}^{K}} F^{(n+1)}=i F_{K}^{(0) I J}\left(\sum_{r=1}^{n} \partial_{I} F^{(r)} \partial_{J} F^{(n+1-r)}-2 \beta D_{I} \partial_{J} F^{(n)}\right),
$$

up to terms of higher order in $N_{(0)}^{-1}$. Here we used that the Levi-Civita connection $D_{I}$ and the non-holomorphic deformation terms of the $F^{(n)}$ involve at least one further power of $N_{(0)}^{-1}$, which can be dropped at the order we are working at. Note that (4.17) is the full holomorphic anomaly equation for the higher $F^{(n)}$ in big moduli space [12]. The standard normalization of the anomaly equation is obtained by setting $-2 \beta=1$.

Let us return to the transformation law (4.13). Inserting the expansion (4.10) into it, we see that $f^{(1)}$ remains invariant under symplectic transformations. This is not what happens in topological string theory, where $f^{(1)}$ transforms into $f^{(1)} \rightarrow f^{(1)}+\alpha \ln \operatorname{det} \mathcal{S}_{0}$, with $\alpha \in \mathbb{R}$. This transformation behavior is, in turn, compensated for by the presence of an additional term $\ln \operatorname{det} N_{I J}^{(0)}$, which ensures that the topological free energy $F^{(1)}$, given by

$$
F^{(1)}(\mathcal{Y}, \overline{\mathcal{Y}})=2 i\left(f^{(1)}(\mathcal{Y})+\bar{f}^{(1)}(\overline{\mathcal{Y}})+\alpha \ln \operatorname{det} N_{I J}^{(0)}\right),
$$

is invariant under symplectic transformations. If we now insist that $F^{(1)}$ and $\Omega$ are related by $F^{(1)}=\left.2 i \Omega_{\Upsilon}\right|_{\Upsilon=0}$, then this is only possible if we take $\Upsilon$ to be real, in which case $\Omega$ given in (4.9) gets modified to

$$
\Omega(Y, \bar{Y}, \Upsilon)=\left(f(Y, \Upsilon)+2 \beta \Upsilon N_{(0)}^{I J} f_{I J}(Y, \Upsilon)+\text { c.c. }\right)+\alpha \Upsilon \ln \operatorname{det} N_{I J}^{(0)}
$$

to first order in $\alpha$. Thus, while the deformation $\beta$ did not enforce any restriction on $\Upsilon$, the presence of the deformation $\alpha$ does.

So far, we restricted ourselves to working at first order in $\alpha, \beta$ and $N_{(0)}^{-1}$. At higher order, the analysis in [12] shows that $\alpha$ and $\beta$ get locked onto the same value $\alpha=\beta$. This is a consequence of the requirement that $\Omega$ should transform consistently under symplectic transformations. In this way we recover the non-holomorphic deformation relevant for the topological string.

\subsection{From Hessian structure to the full anomaly equation}

We now show how to recover the holomorphic anomaly equation (4.17) from the underlying Hessian structure. We proceed as in subsection 3.6, and consider the totally symmetric rank three tensor $S=\nabla g^{H}$, where $g^{H}$ denotes the Hessian metric computed in subsection 4.2. As before, we consider the components

$$
S_{x^{I} \Upsilon}=S_{\Upsilon x^{I} \Upsilon}
$$

Since $S$ is a tensor, we can evaluate this relation in other coordinate systems, in particular in complex supergravity coordinates. As before, using

$$
\begin{aligned}
g_{\Upsilon \Upsilon}^{H} & =-i D_{\Upsilon} F_{\Upsilon}, \\
S_{x^{I} \Upsilon \Upsilon} & =\left.\partial_{x^{I}}\right|_{y} g_{\Upsilon \Upsilon}^{H}=\left.\partial_{x^{I}}\right|_{u} g_{\Upsilon \Upsilon}^{H}+\frac{\partial u^{K}}{\partial x^{I}} \frac{\partial g_{\Upsilon \Upsilon}^{H}}{\partial u^{K}}, \quad \text { where }\left.\partial_{x^{I}}\right|_{u}=\frac{\partial}{\partial Y^{I}}+\frac{\partial}{\partial \bar{Y}^{I}}, \\
S_{\Upsilon x^{I} \Upsilon} & =\left.\frac{\partial g_{x^{I} \Upsilon}^{H}}{\partial \Upsilon}\right|_{x, y}=\left.\frac{\partial g_{x^{I} \Upsilon}^{H}}{\partial \Upsilon}\right|_{x, u}+\frac{\partial u^{K}}{\partial x^{I}} \frac{\partial g_{x^{I} \Upsilon}^{H}}{\partial u^{K}},
\end{aligned}
$$


we obtain, after some rearrangements, an expression for the antiholomorphic derivative

$$
\frac{\partial}{\partial \bar{Y}^{I}} g_{\Upsilon \Upsilon}^{H}=\left.\frac{\partial g_{x^{I} \Upsilon}^{H}}{\partial \Upsilon}\right|_{u}+\frac{\partial u^{K}}{\partial \Upsilon} \frac{\partial g_{x^{I} \Upsilon}^{H}}{\partial u^{K}}-\frac{\partial g_{\Upsilon \Upsilon}^{H}}{\partial Y^{I}}-\frac{\partial u^{K}}{\partial x^{I}} \frac{\partial g_{\Upsilon \Upsilon}^{H}}{\partial u^{K}} .
$$

After a lengthy but straightforward calculation similar to the one in subsection 3.6, we find

$$
\begin{aligned}
-i \frac{\partial}{\partial \bar{Y}^{I}} D_{\Upsilon} F_{\Upsilon}= & \frac{\partial g_{\Upsilon \Upsilon}^{H}}{\partial \bar{Y}^{I}} \\
= & i \bar{F}_{\bar{I} \Upsilon \Upsilon}+2\left(F_{S \Upsilon}+\bar{F}_{\bar{S} \Upsilon}\right) N_{-}^{S L}\left[\bar{F}_{\bar{I} \bar{L} \Upsilon}-\bar{F}_{\bar{I} L \Upsilon}\right] \\
& -i\left(F_{S \Upsilon}+\bar{F}_{\bar{S} \Upsilon}\right) N_{-}^{S L}\left(F_{Q \Upsilon}+\bar{F}_{\bar{Q} \Upsilon}\right) N_{-}^{Q P}\left[\bar{F}_{\bar{I} \bar{P} \bar{L}}+\bar{F}_{\bar{I} P L}-2 \bar{F}_{\bar{I} \bar{P} L}\right]
\end{aligned}
$$

In the holomorphic case, this reduces to (3.17). In the non-holomorphic case based on (4.9) it can be readily verified that when setting $\Upsilon=0$, one obtains the holomorphic anomaly equation (4.17) for $F^{(2)}$ to leading order in $\beta$ and $N_{(0)}^{-1}$. Namely, using (4.9) and setting $\Upsilon=0,(4.19)$ reduces to

$$
\left.\frac{\partial}{\partial \bar{Y}^{I}} D_{\Upsilon} F_{\Upsilon}\right|_{\Upsilon=0}=2 \frac{\partial}{\partial \overline{\mathcal{Y}}^{I}} F^{(2)}=\left.\left(-\bar{F}_{\bar{I} \Upsilon \Upsilon}+F_{S \Upsilon} N_{-}^{S L} F_{Q \Upsilon} N_{-}^{Q P} \bar{F}_{\bar{I} \bar{P} \bar{L}}\right)\right|_{\Upsilon=0} .
$$

Using $\bar{F}_{\bar{I} \Upsilon \Upsilon}\left|\Upsilon=0=-2 i \Omega_{\bar{I} \Upsilon \Upsilon}\right| \Upsilon=0=-8 \beta \bar{F}_{\bar{I}}^{(0) K L} f_{K L}^{(1)}=4 i \beta \bar{F}_{\bar{I}}^{(0) K L} F_{K L}^{(1)}$, and redefining $F^{(n)} \rightarrow 2 i F^{(n)}$, we obtain (4.17) for $F^{(2)}$.

We note that, in principle, one may now proceed to derive the holomorphic anomaly equation for the higher $F^{(n)}$ (with $n \geq 3$ ) by applying covariant derivatives $D_{\Upsilon}$ to (4.19), and subsequently setting $\Upsilon=0$, as in subsection 3.6.

We finish by verifying that the component $S_{x^{I} \Upsilon \bar{\Upsilon}}=\partial_{x^{I}} g_{\Upsilon \bar{\Upsilon}}^{H}$, which is constructed out of the metric component $g_{\Upsilon \bar{\Upsilon}}^{H}=-i D_{\Upsilon} F_{\bar{\Upsilon}}$, does not give rise to an additional non-trivial differential equation. Evaluating the relation $S_{x^{I} \Upsilon \bar{\Upsilon}}=S_{\bar{\Upsilon}_{x^{I} \Upsilon}}=\partial_{\bar{\Upsilon}} g_{x^{I} \Upsilon}^{H}$ in supergravity variables we find that it is identically satisfied. Thus, the only non-trivial differential equation resulting from $g_{\Upsilon \Upsilon}^{H}$ and $g_{\Upsilon \Upsilon}^{H}$ is encoded in the relation $S_{x^{I} \Upsilon \Upsilon}=S_{\Upsilon x^{I} \Upsilon}$.

\section{Concluding remarks}

Let us conclude with a comparison of the approach taken in [12] and the one taken here for obtaining the holomorphic anomaly equation. Both are based on the Hesse potential, which is obtained by a Legendre transform, see (4.5). In the approach of [12] one works directly with the Hesse potential, while here we work with the associated Hessian structure $\left(g^{H}, \nabla\right)$ on the extended scalar manifold $\hat{M}=M \times \mathbb{C}$. The Hesse potential, which is akin to a Hamiltonian [12], is a symplectic function of the special real coordinates. It can also be expressed either in terms of supergravity variables $\left(Y^{I}, \Upsilon\right.$ ) (and their complex conjugate) or in terms of stringy (or covariant) variables $\left(\mathcal{Y}^{I}, \Upsilon\right.$ ) (and their complex conjugate), see (3.7). The approach in [12] consisted in first expressing the Hesse potential in terms of covariant variables by means of a power series expansion in $\Delta Y^{I}$, and then expressing $\Delta Y^{I}$ in terms of a power series in derivatives of $\Omega$, which we recall was introduced in (4.2). In this way it was shown in [12] that the Hesse potential, when expressed in terms of covariant variables, 
equals an infinite sum of symplectic functions, which were denoted by $\mathcal{H}_{i}^{(a)}$. The label $a$ indicates that the leading term is of order $\Omega^{a}$. For higher values of $a$ there are several functions (labelled by $i=1,2, \ldots$ ) with the same value of $a$. This decomposition is unique. In this decomposition there is only one function, namely $\mathcal{H}^{(1)}$, whose leading term is $\Omega$ itself, while the leading term of all the other $\mathcal{H}_{i}^{(a)}$ (with $a \geq 2$ ) involves derivatives of $\Omega$. In [12] it was shown that $\mathcal{H}^{(1)}$ comprises a subsector of the full Hesse potential that encodes the holomorphic anomaly equation. This was achieved by using the explicit expression for $\mathcal{H}^{(1)}$, which consists of an infinite sum that starts with $4 \Omega$, and that involves terms of higher and higher powers of derivatives of $\Omega$. By using the fact that $\Omega$ depends on $\Upsilon$, this infinite sum was in turn rewritten as a series expansion in $\Upsilon$, with coefficient functions that are again symplectic functions. When $\Omega$ is taken to be harmonic, these symplectic functions, denoted by $F^{(n)}$ (the first three of which we display in (C.3)), satisfy the holomorphic anomaly equation (1.1) with $\alpha=0$. Subsequently, by deforming $\Omega$ by $\alpha$-dependent terms, as in (4.18), it was shown [12] that the resulting functions $F^{(n)}$ satisfy the full holomorphic anomaly equation (1.1).

Thus, summarizing, it was shown in [12] that the full Hesse potential, when expressed in terms of covariant variables, contains a subsector $\mathcal{H}^{(1)}$ that, in turn, contains an infinite set of functions $F^{(n)}$ that satisfy the full holomorphic anomaly equation (1.1). The other sectors, described by the other functions $\mathcal{H}_{i}^{(a)}$, are constructed out of derivatives of $\Omega$, and thus contain derived information. They are nevertheless important, since they are needed to build up the full Hesse potential.

In the approach taken in this paper, we instead work with the Hessian metric $g^{H}$ associated with the full Hesse potential. We work in supergravity variables, and we focus on particular components of $g^{H}$, namely on $g_{\Upsilon \Upsilon}^{H}$ and $g_{\Upsilon \bar{\Upsilon}}^{H}$. These two components (both of which are given in terms of the symplectic covariant derivative introduced in $[9,13])$ encode different information. For instance, when evaluated at $\Upsilon=0, g_{\Upsilon \Upsilon}^{H}$ gives the symplectic function $F^{(2)}$, while $g_{\Upsilon \bar{\Upsilon}}^{H}$ gives the symplectic function $\mathcal{H}^{(2)}$ evaluated at $\Upsilon=0$. This is reminiscent of the decomposition of the full Hesse potential into symplectic functions $\mathcal{H}_{i}^{(a)}$ discussed above. We then consider the totally symmetric rank three tensor $S=\nabla g^{H}$, and we first focus on its component $S_{x^{I} \Upsilon \Upsilon}=\partial_{x^{I}} g_{\Upsilon \Upsilon}^{H}$. Evaluating the relation $S_{x^{I} \Upsilon \Upsilon}=$ $S_{\Upsilon x^{I} \Upsilon}$ in supergravity variables and subsequently setting $\Upsilon=0$ we obtain the holomorphic anomaly equation for $F^{(2)}$. One may then ask whether other components of $S$ will lead to additional non-trivial differential equations. To address this, we consider the component $S_{x^{I} \Upsilon \bar{\Upsilon}}=\partial_{x^{I}} g_{\Upsilon \bar{\Upsilon}}^{H}$. Evaluating the relation $S_{x^{I} \Upsilon \bar{\Upsilon}}=S_{\bar{\Upsilon} x^{I} \Upsilon}=\partial_{\bar{\Upsilon}} g_{x^{I} \Upsilon}^{H}$ in supergravity variables we find that it is identically satisfied. Thus, we conclude that the only non-trivial

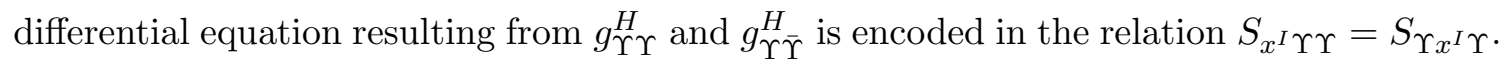

\section{Acknowledgments}

We would like to thank Vicente Cortés and Bernard de Wit for valuable discussions. The work of G.L.C. was partially supported by FCT/Portugal through UID/MAT/04459/2013 and through EXCL/MAT-GEO/0222/2012. The work of T.M. was, in part, supported by STFC and by a BCC visiting fellowship associated to the excellency grant EXCL/MAT- 
GEO/0222/2012. This work was also supported by the COST action MP1210 "The String Theory Universe".

T.M. would like to thank CAMGSD (Department of Mathematics, IST) for the BCC fellowship which enabled him to visit IST, and for the kind hospitality. G.L.C. would like to thank the Max-Planck-Institut für Gravitationsphysik (Albert-Einstein-Institute) for kind hospitality during the completion of this work.

\section{A Connections on vector bundles}

We review some standard facts about connections in vector bundles. Let $E \rightarrow M$ be a vector bundle over a manifold $M$. Then a connection or covariant derivative $\nabla$ on $E$ is a map

$$
\nabla: \mathfrak{X}(M) \times \Gamma(E) \rightarrow \Gamma(E), \quad(X, s) \mapsto \nabla_{X} s
$$

which is a linear derivation (satisfies the product rule) with respect to sections $s \in \Gamma(E)$ of $E$, and which is $C^{\infty}(M)$-linear with respect to vector fields in $X \in \mathfrak{X}(M)$.

For vector bundles of the form $\Omega^{p}(M, E)=\Lambda^{p} T^{*} M \otimes E$, that is for bundles of $p$-forms with values in a vector bundle $E$, the covariant exterior derivative

$$
d_{\nabla}: \Omega^{p}(M, E) \rightarrow \Omega^{p+1}(M, E)
$$

is uniquely determined by its action on sections of $E$. For a given basis $\left\{s_{a}\right\}$ of sections one sets

$$
d_{\nabla} s_{a}=\nabla s_{a}=\omega_{a}^{b} s_{b},
$$

where $\omega_{a}^{b}$ is the connection one-form of $\nabla$. The derivative of a general section $s=f^{a} s_{a} \in$ $\Omega^{0}(M, E)$, where $f^{a} \in C^{\infty}(M)$ is then

$$
d_{\nabla} s=d f^{a} \otimes s_{a}+f^{a} \omega_{a}^{b} s_{b} .
$$

The extension to forms of degree $p>0$ is completely determined by linearity and the product rule

$$
d_{\nabla}(\alpha \otimes s)=d \alpha \otimes s+(-1)^{\operatorname{deg} \alpha} \alpha \wedge d_{\nabla} s,
$$

where $\alpha \in \Omega^{p}(M)$. The exterior covariant derivative $d \nabla$ of $\omega \in \Omega^{p}(M, E)$ can be expressed in terms of the covariant derivative $\nabla$ by

$$
\begin{aligned}
\left(d_{\nabla} \omega\right)\left(X_{0}, \ldots, X_{p}\right)=\sum_{l=0}^{p} & (-1)^{l} \nabla_{X_{l}}\left(\omega\left(\ldots \hat{X}_{l} \ldots\right)\right) \\
& +\sum_{i<j}(-1)^{i+j} \omega\left(\left[X_{i}, X_{j}\right], \ldots \hat{X}_{i} \ldots \hat{X}_{j} \ldots\right),
\end{aligned}
$$

where $X_{0}, \ldots X_{p}$ are vector fields, and where $\hat{X}$ indicates that the corresponding vector field is omitted.

The curvature of the connection $\nabla$ is given by $R^{\nabla}(s)=d_{\nabla}\left(d_{\nabla} s\right)$. If the connection $\nabla$ is flat, then $d_{\nabla}^{2}=0$, so that $d_{\nabla}$ defines an exact sequence. In this case a version of the Poincaré lemma exists. 


\section{B Special coordinates}

One of the defining conditions of affine special Kähler geometry is $d_{\nabla} J=0$, where $J$ is the complex structure. We can apply the above results since $J$ is a section of $\operatorname{End}(E) \simeq E^{*} \otimes E$, where $E=T M$. In the following we derive the local form (2.2) of (2.1), and also explain how the existence of special coordinates can be derived.

Let $A$ be a section of $\operatorname{End}(T M)$. Choosing dual bases $\left\{e^{a}\right\},\left\{e_{a}\right\}$ of sections, and regarding $A$ as a $T M$-valued one-form, we have

$$
A=A^{a} e_{a}=A_{b}^{a} e^{b} \otimes e_{a} \in \Omega^{1}(M, T M)
$$

and

$$
d_{\nabla} A=d A^{a} \otimes e_{a}-A^{a} \wedge \omega_{a}^{b} e_{b} \in \Omega^{2}(M, T M) .
$$

If the connection $\nabla$ is flat, we can choose sections $e_{a}$ such that the connection one-form vanishes, $\omega_{a}^{b}=0$ and in such a frame $d_{\nabla} A=0$ reduces to $d A^{a}=0$. Thus the one-forms $A^{a}$ are locally exact, $A^{a}=d \phi^{a}$.

Since $E=T M$, the torsion of the connection $\nabla$ is defined by

$$
T^{\nabla}(X, Y)=\nabla_{X} Y-\nabla_{Y} X-[X, Y]
$$

One can show that

$$
T^{\nabla}(X, Y)=d_{\nabla} \operatorname{Id}(X, Y)
$$

where

$$
\mathrm{Id}=e^{a} \otimes e_{a}=\delta_{a}^{b} e^{a} \otimes e_{b}
$$

is the identity endomorpism of $T M$. If $\nabla$ is both flat and torsion free, then $d_{\nabla} \mathrm{Id}=0$ implies that in a frame where the connection vanishes, the one-forms $I^{a}=e^{a}$ are locally exact $e^{a}=d t^{a}$. This defines a set of $\nabla$-affine coordinates. In such coordinates the condition (2.1) becomes

$$
d_{\nabla} J=0 \Rightarrow d J^{a}=0 \Rightarrow \partial_{[b} J_{c]}^{b}=0 .
$$

If the manifold $M$ is in addition equipped with a non-degenerate, closed two-form $\omega \in \Omega^{2}(M), d \omega=0$, then a connection $\nabla$ is called symplectic if the symplectic form $\omega$ is parallel:

$$
\nabla \omega=0 \Leftrightarrow \nabla_{X} \omega=X\left(\omega_{a b}\right) e^{a} \wedge e^{b}+\omega_{a b}\left(\nabla_{X} e^{a}\right) \wedge e^{b}+\omega_{a b} e^{a} \wedge \nabla_{X} e^{b}=0
$$

for all vector fields $X$. If the connection $\nabla$ is in addition flat, we can choose sections $e^{a}$ such that $\nabla_{X} e^{a}=0$. With respect to such a basis the coefficients of $\omega$ are constant, $X\left(\omega_{a b}\right)=0$. If the connection $\nabla$ is in addition torsion-free, the co-frame $e^{a}$ comes from an affine coordinate system $t^{a}$, and

$$
\omega=\frac{1}{2} \omega_{a b} d t^{a} \wedge d t^{b}
$$


where $\omega_{a b}$ is a constant, antisymmetric, non-degenerate matrix. Using the linear part of the affine transformation that we can apply to $t^{a}$, the matrix $\omega_{a b}$ can be brought to the standard form

$$
\left(\omega_{a b}\right)^{\text {Standard }}=\left(\begin{array}{cc}
0 & \mathbb{1} \\
-\mathbb{1} & 0
\end{array}\right) .
$$

This form is still invariant under affine transformations where the linear part is symplectic. The associated coordinates are called Darboux coordinates. Thus we have seen that for a flat, torsion-free, symplectic connection the $\nabla$-affine coordinates can be chosen to be Darboux coordinates. In the context of affine special Kähler geometry such coordinates are called special real coordinates.

We remark that in the main part of this paper we use special real coordinates $\left(q^{a}\right)=$ $\left(x^{I}, y_{I}\right)$ where the Kähler form takes the form

$$
\omega=2 d x^{I} \wedge d y_{I}=\Omega_{a b} d q^{a} \wedge d q^{b} .
$$

Note that the components of $\omega$ with respect to the coordinates $\left(q^{a}\right)$ are $\omega_{a b}=2 \Omega_{a b}=$ $2 \omega_{a b}^{\text {Standard }}$. In other words the special real coordinates differ from standard Darboux coordinates by a conventional factor $\sqrt{2}$.

\section{Symplectic transformations and functions}

Symplectic transformations acts as follows on a symplectic vector $\left(Y^{I}, F_{I}\right)(I=0, \ldots n)$,

$$
\begin{aligned}
Y^{I} & \rightarrow U^{I}{ }_{J} Y^{J}+Z^{I J} F_{J}, \\
F_{I} & \rightarrow V_{I}{ }^{J} F_{J}+W_{I J} Y^{J},
\end{aligned}
$$

where $U, V, Z, W$ are the $(n+1) \times(n+1)$ real submatrices that give rise to an element of $\operatorname{Sp}(2 n+2, \mathbb{R})$. When $F$ equals the prepotential $F^{(0)}$, then $N_{(0)}^{I J}$ transforms as follows under symplectic transformations [9],

$$
N_{(0)}^{I J} \rightarrow \overline{\mathcal{S}}_{0}{ }^{I}{ }_{K} \mathcal{S}_{0}{ }^{J}{ }_{L} N_{(0)}^{K L}=\mathcal{S}_{0}{ }^{I}{ }_{K} \mathcal{S}_{0}{ }^{J}{ }_{L}\left(N_{(0)}^{K L}-i \mathcal{Z}_{0}^{K L}\right),
$$

where

$$
\begin{aligned}
\mathcal{S}_{0}{ }^{I} K & =U^{I}{ }_{K}+Z^{I J} F_{J K}^{(0)}, \\
\mathcal{Z}_{0}^{I J} & =\left[\mathcal{S}_{0}^{-1}\right]_{K}^{I} Z^{K J} .
\end{aligned}
$$

Consider a generalized prepotential $F(Y, \bar{Y}, \Upsilon, \bar{\Upsilon})=F^{(0)}(Y)+2 i \Omega(Y, \bar{Y}, \Upsilon, \bar{\Upsilon})$, where $\Omega$ is taken to be harmonic, $\Omega(Y, \bar{Y}, \Upsilon, \bar{\Upsilon})=f(Y, \Upsilon)+\bar{f}(\bar{Y}, \bar{\Upsilon})$. Expanding $f(Y, \Upsilon)$ as in (4.10) and inserting this into (3.12), yields explicit expressions for the symplectic functions $F^{(n)}$. The first three read as follows,

$$
\begin{aligned}
& F^{(1)}=2 i f^{(1)} \\
& F^{(2)}=2 i\left(f^{(2)}-N_{(0)}^{I J} f_{I}^{(1)} f_{J}^{(1)}\right) \\
& F^{(3)}=2 i\left(f^{(3)}-2 N_{(0)}^{I J} f_{I}^{(2)} f_{J}^{(1)}+2 f_{I}^{(1)} N_{(0)}^{I J} f_{J K}^{(1)} N_{(0)}^{K L} f_{L}^{(1)}\right. \\
& \left.\quad+\frac{2 i}{3} F_{I J K}^{(0)} N_{(0)}^{I P} N_{(0)}^{J Q} N_{(0)}^{K R} f_{P}^{(1)} f_{Q}^{(1)} f_{R}^{(1)}\right),
\end{aligned}
$$


in accordance with [12]. These expressions get modified when $\Omega$ is not any longer harmonic. The resulting expressions for the topological string can be found in appendix D of [12].

Open Access. This article is distributed under the terms of the Creative Commons Attribution License (CC-BY 4.0), which permits any use, distribution and reproduction in any medium, provided the original author(s) and source are credited.

\section{References}

[1] B. de Wit and A. Van Proeyen, Potentials and symmetries of general gauged $N=2$ supergravity: Yang-Mills models, Nucl. Phys. B 245 (1984) 89 [INSPIRE].

[2] B. de Wit, P.G. Lauwers and A. Van Proeyen, Lagrangians of $N=2$ supergravity-matter systems, Nucl. Phys. B 255 (1985) 569 [INSPIRE].

[3] A. Strominger, Special geometry, Commun. Math. Phys. 133 (1990) 163 [InSPIRE].

[4] L. Castellani, R. D'Auria and S. Ferrara, Special Kähler geometry: an intrinsic formulation from $N=2$ space-time supersymmetry, Phys. Lett. B 241 (1990) 57 [INSPIRE].

[5] L. Castellani, R. D'Auria and S. Ferrara, Special geometry without special coordinates, Class. Quant. Grav. 7 (1990) 1767 [INSPIRE].

[6] D.S. Freed, Special Kähler manifolds, Commun. Math. Phys. 203 (1999) 31 [hep-th/9712042] [INSPIRE].

[7] D.V. Alekseevsky, V. Cortes and C. Devchand, Special complex manifolds, J. Geom. Phys. 42 (2002) 85 [math/9910091] [INSPIRE].

[8] V. Cortés, A holomorphic representation formula for parabolic hyperspheres, in the proceedings of the International Conference "PDEs, Submanifolds and Affine Differential Geometry" (Warsaw 2000), B. Opozda et al. eds., Banach Center Publications, Poland (2001).

[9] B. de Wit, $N=2$ electric-magnetic duality in a chiral background, Nucl. Phys. Proc. Suppl. 49 (1996) 191 [hep-th/9602060] [INSPIRE].

[10] M. Bershadsky, S. Cecotti, H. Ooguri and C. Vafa, Kodaira-Spencer theory of gravity and exact results for quantum string amplitudes, Commun. Math. Phys. 165 (1994) 311 [hep-th/9309140] [INSPIRE].

[11] I. Antoniadis, E. Gava, K.S. Narain and T.R. Taylor, Topological amplitudes in string theory, Nucl. Phys. B 413 (1994) 162 [hep-th/9307158] [INSPIRE].

[12] G.L. Cardoso, B. de Wit and S. Mahapatra, Deformations of special geometry: in search of the topological string, JHEP 09 (2014) 096 [arXiv:1406.5478] [INSPIRE].

[13] G.L. Cardoso, B. de Wit and S. Mahapatra, Non-holomorphic deformations of special geometry and their applications, Springer Proc. Phys. 144 (2013) 1 [arXiv:1206.0577] [INSPIRE].

[14] A. Ceresole, R. D'Auria, S. Ferrara and A. Van Proeyen, Duality transformations in supersymmetric Yang-Mills theories coupled to supergravity, Nucl. Phys. B 444 (1995) 92 [hep-th/9502072] [INSPIRE].

[15] T. Mohaupt, Black hole entropy, special geometry and strings, Fortsch. Phys. 49 (2001) 3 [hep-th/0007195] [INSPIRE]. 
[16] D.Z. Freedman and A. Van Proeyen, Supergravity, Cambridge University Press, Cambridge U.K. (2012).

[17] G.L. Cardoso and A. Veliz-Osorio, On the $\sigma$-model of deformed special geometry, Nucl. Phys. B 872 (2013) 228 [arXiv:1212.4364] [INSPIRE].

[18] G.L. Cardoso, B. de Wit, J. Kappeli and T. Mohaupt, Black hole partition functions and duality, JHEP 03 (2006) 074 [hep-th/0601108] [INSPIRE]. 